\title{
Preoperative stimulation of resolution and inflammation blockade eradicates micrometastases
}

\author{
Dipak Panigrahy, ${ }^{1,2,3}$ Allison Gartung, ${ }^{1,2,3}$ Jun Yang, ${ }^{4}$ Haixia Yang, ${ }^{1,2,3}$ Molly M. Gilligan, ${ }^{1,2,3}$ Megan L. Sulciner, ${ }^{1,2,3}$ Swati S. Bhasin, ${ }^{5}$ \\ Diane R. Bielenberg, ${ }^{6}$ Jaimie Chang, ${ }^{1,2,3}$ Birgitta A. Schmidt, ${ }^{7}$ Julia Piwowarski, ${ }^{1,2,3}$ Anna Fishbein, ${ }^{1,2,3}$ Dulce Soler-Ferran, ${ }^{1,2,3}$ \\ Matthew A. Sparks, ${ }^{8}$ Steven J. Staffa, ${ }^{9}$ Vidula Sukhatme, ${ }^{10}$ Bruce D. Hammock, ${ }^{4}$ Mark W. Kieran, ${ }^{11,12}$ Sui Huang, ${ }^{13}$ Manoj Bhasin, ${ }^{5}$ \\ Charles N. Serhan, ${ }^{14}$ and Vikas P. Sukhatme ${ }^{3,5,15}$ \\ 'Center for Vascular Biology Research, ${ }^{2}$ Department of Pathology, and ${ }^{3}$ Cancer Center, Beth Israel Deaconess Medical Center, Harvard Medical School, Boston, Massachusetts, USA. ${ }^{4}$ Department of \\ Entomology and Nematology, and UC Davis Comprehensive Cancer Center, University of California, Davis, California, USA. ${ }^{5}$ Division of Interdisciplinary Medicine and Biotechnology, Department of Medicine, \\ Beth Israel Deaconess Medical Center, Harvard Medical School, Boston, Massachusetts, USA. ${ }^{6}$ ascular Biology Program and 'Department of Pathology, Boston Children's Hospital, Harvard Medical School, \\ Boston, Massachusetts, USA. ${ }^{8}$ Division of Nephrology, Department of Medicine, Duke University and Durham VA Medical Centers, Durham, North Carolina, USA. ${ }^{9}$ Department of Anesthesiology, Critical Care \\ and Pain Medicine, Boston Children's Hospital, Harvard Medical School, Boston, Massachusetts, USA. ${ }^{10}$ ClobalCures Inc., Newton, Massachusetts, USA. "Division of Pediatric Oncology, Dana-Farber Cancer \\ Institute, and ${ }^{12}$ Department of Pediatric Hematology/Oncology, Boston Children's Hospital, Harvard Medical School, Boston, Massachusetts, USA. ${ }^{13}$ Institute for Systems Biology, Seattle, Washington,
} USA. ${ }^{14}$ Center for Experimental Therapeutics and Reperfusion Injury, Department of Anesthesiology, Perioperative and Pain Medicine, Brigham and Women's Hospital, Harvard Medical School, Boston, Massachusetts, USA. ${ }^{15}$ Department of Medicine and Center for Affordable Medical Innovation, Emory University School of Medicine, Atlanta, Georgia, USA.

\begin{abstract}
Cancer therapy is a double-edged sword, as surgery and chemotherapy can induce an inflammatory/immunosuppressive injury response that promotes dormancy escape and tumor recurrence. We hypothesized that these events could be altered by early blockade of the inflammatory cascade and/or by accelerating the resolution of inflammation. Preoperative, but not postoperative, administration of the nonsteroidal antiinflammatory drug ketorolac and/or resolvins, a family of specialized proresolving autacoid mediators, eliminated micrometastases in multiple tumor-resection models, resulting in long-term survival. Ketorolac unleashed anticancer T cell immunity that was augmented by immune checkpoint blockade, negated by adjuvant chemotherapy, and dependent on inhibition of the COX-1/thromboxane $A_{2}\left(T X A_{2}\right)$ pathway. Preoperative stimulation of inflammation resolution via resolvins ( $R v D 2, R v D 3$, and RvD4) inhibited metastases and induced T cell responses. Ketorolac and resolvins exhibited synergistic antitumor activity and prevented surgery- or chemotherapy-induced dormancy escape. Thus, simultaneously blocking the ensuing proinflammatory response and activating endogenous resolution programs before surgery may eliminate micrometastases and reduce tumor recurrence.
\end{abstract}

\section{Introduction}

Cancer treatment is a double-edged sword, as surgery (including biopsy), chemotherapy, or radiation can induce tumor-dormancy escape and subsequent metastatic outgrowth by impairing tumor-specific immunity through inflammation-mediated growth signals and loss of resolution of inflammation (1-16). Even anesthetics can impair inflammation resolution (17). Recent results show that chemotherapy-generated cell death can paradoxically promote tumor growth via the release of proinflammatory and

\section{Related Commentary: p. 2663}

Authorship note: DP, AG, and JY contributed equally to this work. Conflict of interest: BIDMC has filed patents on behalf of DP and VPS on the use of selective COX-1/TXA2 antagonists for preventing cancer recurrence. VPS is a consultant and equity holder in MitraBiotech, Berg, GMDx, and Victa Biotherapeutics. MB is an equity holder at Anxome, BiomaRx, Canomiks, and CMDx. MWK's current position at Bristol-Myers Squibb is not related to this work.

Copyright: (c) 2019, American Society for Clinical Investigation.

Submitted: January 11, 2019; Accepted: April 17, 2019; Published: June 17, 2019

Reference information: J Clin Invest. 2019;129(7):2964-2979.

https://doi.org/10.1172/JCl127282. proangiogenic cytokines $(9,10,18)$. Moreover, a preoperative cycle of chemotherapy can stimulate proinflammatory cytokines after cancer surgery (19), and surgical wounding may impair the efficacy of chemotherapy (20).

In the treatment of locoregional disease, the perioperative period offers a unique window for curbing the risk of metastatic growth and relapse $(2,4,21-27)$. For instance, a bimodal pattern of recurrence for early stage breast and lung cancers suggests that surgery potentiates the metastatic process by inducing tumordormancy escape of micrometastatic lesions (28-30). Micrometastases present in cancer patients at the time of surgery are associated with reduced survival (31). Moreover, surgery can promote metastasis, not simply by mechanical dissemination of cancer cells, but also by stimulation of systemic inflammation and surgery-associated immunosuppression, resulting in outgrowth of dormant cancer cells at distant sites (2).

Over $30 \%$ of healthy individuals harbor microscopic dormant cancers (32), and noncancer surgery and anesthesia may promote the growth of such occult microtumors (33). Importantly, retrospective analyses of tumor recurrence in patients undergoing breast cancer surgery revealed that preoperative administration of ketorolac 
was associated with a marked reduction of recurrence and mortality after surgery (34). However, ketorolac did not exhibit cancer-preventive activity when administered postoperatively, which is when NSAIDs are routinely administered for pain management (34). Preoperative ketorolac increased blood $\mathrm{CD}^{+} \mathrm{T}$ cells in patients undergoing tumor resection, potentially reversing surgery-induced immunosuppression during the perioperative period (35).

Chronic inflammation has been associated with tumor-promoting activity $(36,37)$, in part due to a deficit in the resolution of inflammation (12). Cancer therapies have focused on blocking the production of COX-2-derived eicosanoids to suppress tumor-promoting inflammation (38). However, COX-2 is also host protective, as its metabolite, prostaglandin $\mathrm{E}_{2}\left(\mathrm{PGE}_{2}\right)$, plays a role in the resolution of inflammation in the chronic phase (39). Specifically, tight regulation of the temporal pattern of $\mathrm{PGE}_{2}$ release is critical for activating the class switching of lipid mediators from production of inflammatory mediators to that of proresolution signals through specialized proresolving mediators (SPMs) $(40,41)$. $\mathrm{PGE}_{2}$ released by dead cells negatively regulates an inflammatory response activated by damage-associated molecular patterns, which may also contribute to the proresolution activity of $\mathrm{PGE}_{2}$ (42). Other COX-2-derived prostaglandins of the D2 and J2 series generate lipid mediators that accelerate resolution of inflammation and control endogenous inflammation (12). Thus, COX-2 inhibitors may be "resolution toxic," as they suppress the production of these prostaglandins $(12,39,40,43)$ and may worsen therapy-induced cancer progression.

$\mathrm{PGE}_{2}$ also exhibits immunosuppressive activity, stimulates regulatory $\mathrm{T}$ cells, inhibits antigen presentation, and suppresses NK cells $(36,38)$. Although these functions demonstrate the role of $\mathrm{PGE}_{2}$ in the resolution phase of inflammation, they may inhibit antitumor immunity, hence promoting tumor escape (36). The dual activities of eicosanoids may explain the biphasic dose-dependent or paradoxical relationship between chronic use of NSAIDs and cancer risk (44). Given the opposing roles of eicosanoids, NSAIDs that are intended to block tumor-promoting inflammation may counter the resolution process and thus impose an inherent limitation of efficacy. Thus, simultaneously blocking the proinflammatory response and activating endogenous resolution programs may control cancer therapy-stimulated inflammation.

The SPM superfamily consists of potent immunoresolvent agonists derived from omega-3 fatty acids (e.g., resolvins, protectins, and maresins) as well as arachidonic acid (e.g., lipoxins) (12, $43,45-47)$. Resolvins, lipoxins, and aspirin-triggered SPMs exhibit antitumor activity by promoting the clearance of therapy-generated tumor cell debris and counterregulating proinflammatory cytokines (9, 48-51). Interestingly, unlike other synthetic nonsalicylate NSAIDs, aspirin irreversibly acetylates COX-2 and converts its enzymatic activity to produce aspirin-triggered SPMs. Thus, aspirin's mechanism of action involves both inhibition of proinflammatory mediators and stimulation of proresolving mediators $(12,51)$.

Here, we utilize a well-established animal model in which dormancy escape and outgrowth of lung metastases are triggered by primary tumor resection to study therapeutic approaches to overcoming the tumor-promoting capability of surgery. We show that a single preoperative, but not postoperative, dose of ketorolac suppresses lung micrometastases present at the time of primary tumor resection, an outcome dependent on COX-2 activity and host antitumor immunity as well as inhibition of COX-1-derived thromboxane $\mathrm{A}_{2}\left(\mathrm{TXA}_{2}\right)$. Moreover, preoperative acceleration of inflammation resolution with resolvins inhibited micrometastases and prevented tumor-dormancy escape. Our results indicate that preoperative and perichemotherapeutic interventions can control tumor recurrence via inflammation resolution and promotion of host antitumor immunity.

\section{Results}

Preoperative ketorolac eradicates micrometastases and promotes long-term survival in multiple tumor-resection models. To investigate whether preoperative ketorolac affects survival in tumor-resection models, we utilized a metastatic lung cancer model in which primary syngeneic Lewis lung carcinoma (LLC) tumors were grown to $1500-2000 \mathrm{~mm}^{3}$ in male $\mathrm{C} 57 \mathrm{BL} / 6 \mathrm{~J}$ mice, resulting in micrometastases at the time of tumor resection $(9,52,53)$. Following resection of primary tumors, control mice reproducibly succumbed to lung metastasis by day 24 after resection (Figure 1A). While 60\% of mice administered preoperative ketorolac expired from macroscopic lung metastases by day 43 , the remaining $40 \%$ exhibited long-term survival (defined as $>90$ days after tumor resection). In contrast, postoperative ketorolac did not prolong survival compared with that of control animals, as all postoperative ketorolactreated mice were moribund from spontaneous lung metastasis by day 25 after resection (Figure 1A).

$\mathrm{H} \& \mathrm{E}$ staining revealed abundant micrometastases throughout the lungs at the time of LLC resection (day 0) (Figure 1B). Micrometastases were also detected at 7 days after LLC resection in approximately $60 \%$ of ketorolac-treated mice (Supplemental Figure 1A; supplemental material available online with this article; https://doi. org/10.1172/JCI127282DS1). In contrast, no micrometastases were detected in lungs from preoperative ketorolac-treated long-term survivors (day 240) (Figure 1B). We conducted similar experiments in the highly invasive E0771 and orthotopic 4T1 breast cancer models, which metastasize to the lungs (54). Preoperative ketorolac resulted in long-term survival in $30 \%$ of mice at 240 days after resection compared with control mice in the E0771 model (Supplemental Figure 1B). In an orthotopic $4 \mathrm{~T} 1$ breast cancer model in female $\mathrm{BALB} / \mathrm{cJ}$ mice, preoperative ketorolac resulted in sustained survival in $40 \%$ of these mice after mastectomy (Supplemental Figure 1C). Thus, the antitumor activity of preoperative ketorolac is independent of tumor type, sex, strain, or location of the primary tumor.

Ketorolac prevents surgery- and chemotherapy-induced tumordormancy escape. Systemic tumor recurrence after primary tumor resection can result from stimulation of dormant micrometastases present at the time of surgery $(1,2,52)$, tumor cell dissemination during surgery $(1,55)$, or de novo tumorigenesis. To determine whether ketorolac can suppress surgery- or chemotherapy-induced tumor-dormancy escape, we utilized nonresection models in which mice are injected with a subthreshold (nontumorigenic) inoculum of $10^{4}$ LLC, $10^{4}$ EL4 (lymphoma), or 10 316 F10 (melanoma) tumor cells. Despite the presence of tumor cells, mice in this model can survive for over 200 days without evidence of progressive tumor growth, thereby mimicking tumor dormancy and minimal residual disease $(9,53,56)$. Consistent with surgery-stimulated tumor growth (1-4), laparotomy performed distant from the primary 
A

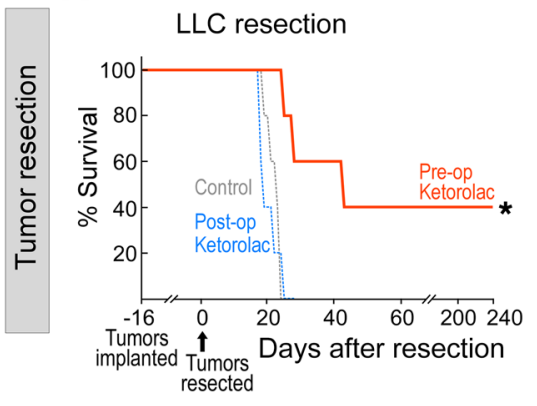

C

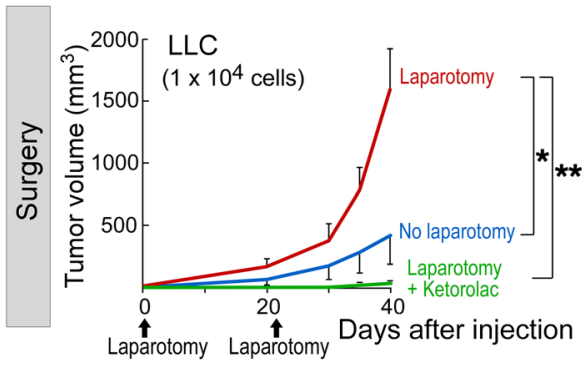

$\mathbf{F}$

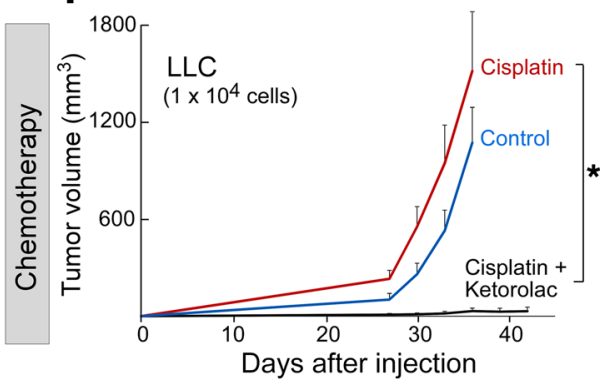

B
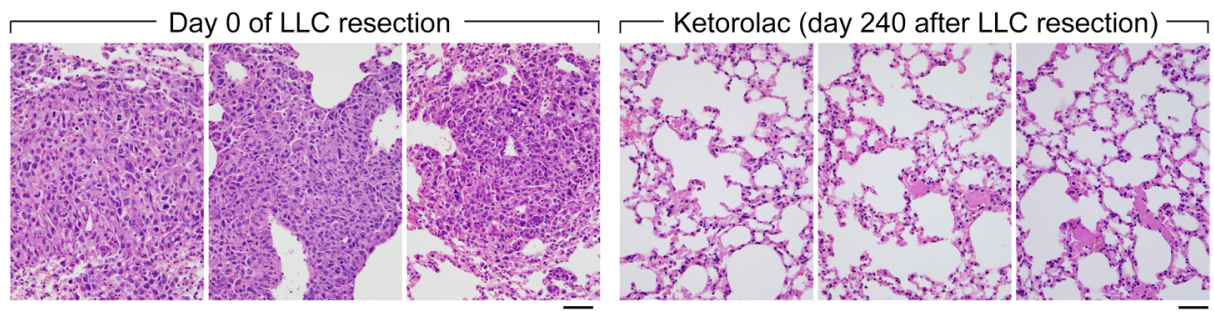

E
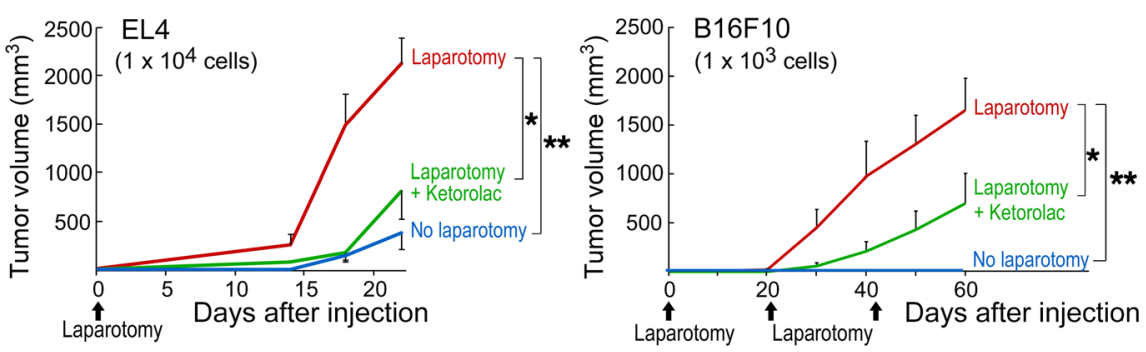

G

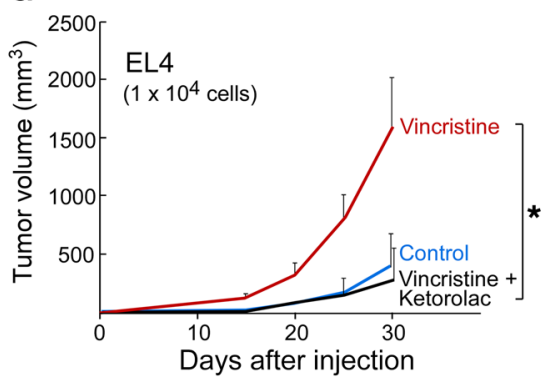

H

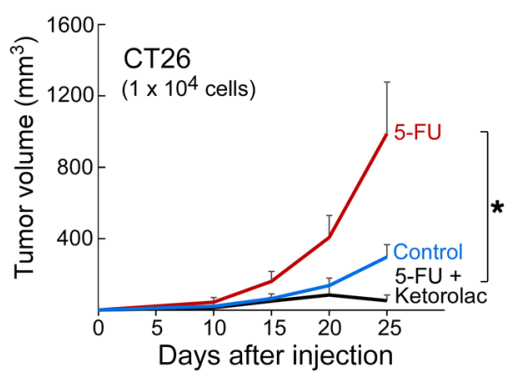

Figure 1. Preoperative ketorolac promotes long-term survival and prevents therapy-induced dormancy escape. (A) Preoperative vs. postoperative ketorolac effects on survival after primary tumor resection in a spontaneous LLC metastasis model. $n=5$ mice/group. Kaplan-Meier analysis log-rank test, ${ }^{*} P<0.01$, control or postoperative ketorolac vs. preoperative ketorolac. (B) H\&E staining of lungs from mice at the time of LLC tumor resection (day 0 ) or from preoperative ketorolac-treated mice at 240 days after resection. Representative micrographs of 10 mice/group. Scale bars: $50 \mu \mathrm{m}$. (C-E) Growth of LLC, EL4, or B16F10 in mice treated with preoperative ketorolac or control subjected to laparotomy (day 0, 21, and/or 42 after injection) vs. no laparotomy. $n=10-20$ mice/group. Two-way repeated measure mixed-effects ANOVAs for tumor growth rates and 2-tailed Student's $t$ test for final tumor measurements were used throughout unless specified. (C) ${ }^{*} P<0.001$, laparotomy vs. no laparotomy; ${ }^{*} P<0.001$, laparotomy and ketorolac vs. laparotomy. (D) ${ }^{*} P=0.009$, laparotomy and ketorolac vs. laparotomy; ${ }^{*} P<0.001$, laparotomy vs. no laparotomy. $(E){ }^{*} P<0.05$, laparotomy and ketorolac vs. laparotomy; ${ }^{*} P<0.05$, laparotomy vs. no laparotomy. (F-H) Growth of LLC, EL4, or CT26 (104 cells) in response to chemotherapy and/or ketorolac. Ketorolac was administered the day before, day of, and day after chemotherapy. Systemic chemotherapy was initiated on day of tumor cell injection. (F) $n=15-28$ mice/ group. ${ }^{*} P<0.001$, cisplatin and ketorolac vs. cisplatin (day 36 after injection). (C) $n=5$ mice/group. ${ }^{*} P<0.05$, control or vincristine and ketorolac vs. vincristine (day 30 after injection). (H) $n=5$ mice/group. ${ }^{*} P<0.01$, control or 5-FU and ketorolac vs. 5-FU (day 25 after injection).

tumor implantation site $\left(10^{4}\right.$ cells) stimulated LLC tumor-dormancy escape (Figure 1C). Preoperative ketorolac suppressed laparotomy-induced dormancy escape in $80 \%$ of mice by day 40 after tumor cell injection (Figure 1C). Similarly, preoperative ketorolac suppressed laparotomy-stimulated EL4 and B16F10 dormancy escape in $40 \%-60 \%$ of mice by day 22 and day 60 after tumor cell injection, respectively (Figure 1, D and E, and Supplemental Figure 1D). Next, we utilized GFP-labeled LLC tumor cells ( $10^{4}$ cells) to monitor the impact of preoperative ketorolac on dormant tumor cells in mice subjected to laparotomy. Remarkably, LLC-GFP tumor cells were not detected at the tumor implantation site after laparotomy in preoperative ketorolac-treated mice (Supplemental Figure $1 \mathrm{E})$, suggesting that ketorolac eliminated dormant tumor cells.
While chemotherapeutic agents can suppress growth of established tumors, they can paradoxically stimulate the growth of a subthreshold inoculum of the identical tumor type $(9,18)$. Perichemotherapeutic administration of ketorolac neutralized chemotherapy-stimulated tumor growth in mice injected with a subthreshold inoculum of $10^{4}$ tumor cells, including cisplatinstimulated LLC, vincristine-stimulated EL4, and 5-fluorouracilstimulated (5-FU-stimulated) CT26 (colon carcinoma) (Figure 1, F-H). However, GFP-labeled tumor cells were detected at the tumor implantation site when ketorolac was administered with cisplatin to mice bearing LLC-GFP tumors (Supplemental Figure $1 F)$. Thus, ketorolac inhibited both surgery- and chemotherapyinduced tumor-dormancy escape. 
A LLC resection (COX-1 inhibitors)

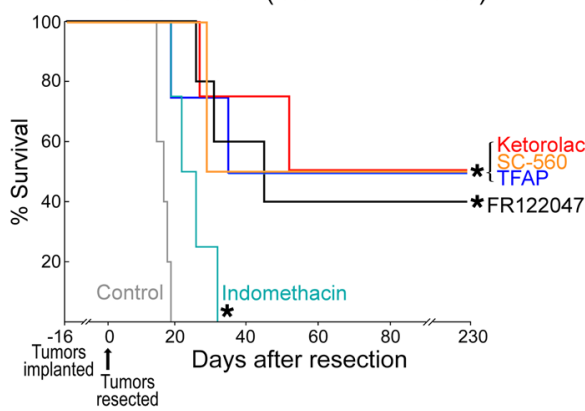

B LLC resection (NSAIDs)

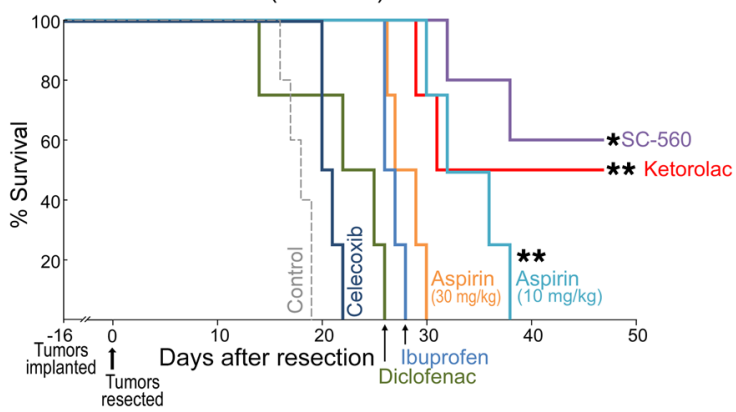

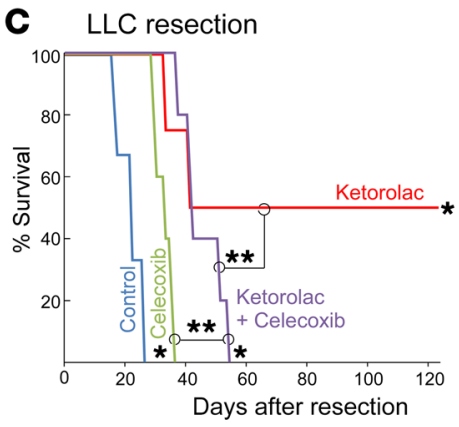

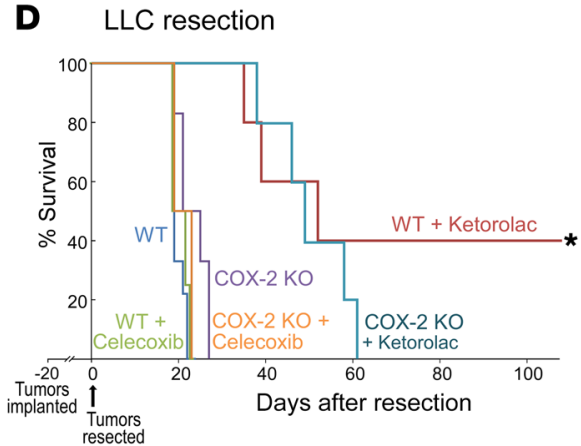

E LLC resection

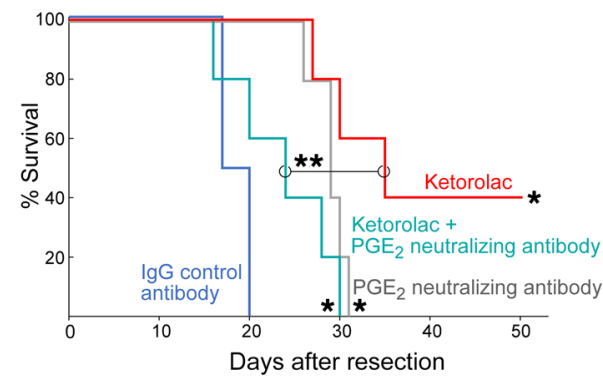

Figure 2. COX-1 inhibition and baseline COX-2 activity are critical for the antitumor activity of ketorolac. (A) Preoperative COX-1 inhibitors (FR122047, TFAP, or SC-560), ketorolac, or nonselective COX inhibitor (indomethacin) effects on survival after LLC resection. $n=4-6$ mice/group. ${ }^{*} P<0.05$, FR122047, TFAP, SC-560, ketorolac, or indomethacin vs. control. (B) Preoperative NSAIDs effect on survival after LLC resection. $n=4-5$ mice/group. ${ }^{*} P=0.003$, SC-560 vs. control; ${ }^{* *} P<0.05$, ketorolac or aspirin $(10 \mathrm{mg} / \mathrm{kg})$ vs. control. (C) Preoperative celecoxib and/or ketorolac effects on survival after LLC resection. $n=4-6$ mice/group. ${ }^{*} P<0.05$, ketorolac, celecoxib, or ketorolac and celecoxib vs. control. ${ }^{* *} P<0.05$ ketorolac or celecoxib vs. ketorolac and celecoxib. (D) Preoperative ketorolac vs. celecoxib effects on survival after LLC resection in WT or COX-2-KO mice. $n=4-9$ mice/group. ${ }^{*} P<0.05$, WT ketorolac vs. COX-2 KO ketorolac. (E) Preoperative ketorolac and/or PGE depletion effects on survival after LLC resection. $n=5-6$ mice/group. ${ }^{*} P<0.05$, ketorolac, $P G E_{2}$ neutralizing antibody, or ketorolac and $\mathrm{PGE}_{2}$ neutralizing antibody vs. control. ${ }^{* *} P<0.01$, ketorolac vs. ketorolac and $P G E_{2}$ neutralizing antibody.

COX-1/TXA inhibition and basal COX-2 activity are critical for the antitumor activity of ketorolac. Among the FDA-approved NSAIDs, ketorolac preferentially inhibits COX-1 and exhibits lower COX-2 activity $(57,58)$. To determine whether the observed antitumor activity of preoperative ketorolac was mediated by COX-1 and/or COX-2 inhibition, we utilized 3 highly selective COX-1 inhibitors (SC-560, FR122047, or TFAP), the selective COX-2 inhibitor celecoxib, and the nonselective COX inhibitor indomethacin $(38,59)$. Similar to ketorolac, preoperative administration of the selective COX-1 inhibitors resulted in long-term survival in $40 \%-50 \%$ of mice up to 230 days after LLC resection (Figure 2A). However, celecoxib did not result in sustained survival (Figure 2B), suggesting that the observed antitumor activity of ketorolac is likely mediated by COX-1 inhibition.

Although indomethacin prolonged survival compared with control, no long-term survivors after LLC resection were noted (Figure 2A). A similar response profile of antitumor activity was observed with other nonselective NSAIDs, such as diclofenac, ibuprofen, and high-dose aspirin ( $30 \mathrm{mg} / \mathrm{kg}$ ) (Figure 2B). Aspirin can prevent metastasis by inhibiting COX-1 activity and subsequent TXA synthesis $(60,61)$. Low-dose aspirin prolonged survival compared with high-dose aspirin (which engenders more complete inhibition of COX-2) (Figure 2B), suggesting that preoperative COX-2 inhibition may negate the anticancer activity of ketorolac. Indeed, preoperative coadministration of celecoxib and ketorolac resulted in no long-term survivors (Figure 2C). To confirm that COX-2 inhibition may impair the activity of preoperative ketorolac, we next performed the LLC tumor-resection experiments in COX-2-KO mice. While preoperative ketorolac resulted in long-term survival after resection in WT mice, long-term survival was not observed in COX-2-KO mice (Figure 2D). Moreover, the combination of preoperative ketorolac and an anti- $\mathrm{PGE}_{2}$ neutralizing antibody did not result in any long-term survivors after resection (Figure 2E). Therefore, in the LLC tumor-resection model, baseline COX-2 activity and $\mathrm{PGE}_{2}$ levels may be necessary for the antitumor activity of ketorolac.

Since COX-1 preferentially mediates TXA ${ }_{2}$ production $(38$, 61), we measured plasma $\mathrm{TXB}_{2}$, a stable hydration product of $\mathrm{TXA}_{2}$ used to assess COX-1 activity. Profiling based on liquid chromatography-tandem mass spectrometry (LC-MS/MS) revealed dramatic reduction of $\mathrm{TXB}_{2}$ at 2 hours after LLC resection as well as a lesser reduction in $\mathrm{PGE}_{2}$ in ketorolac-treated mice compared with control (Figure 3, A and B). To ascertain a functional role for TXA $_{2}$ in the observed antitumor activity of ketorolac, we performed primary tumor resections in mice lacking the thromboxane prostanoid (TP) receptor. TP-KO mice exhibited long-term survival compared with WT mice (Figure 3C). Moreover, preoperative administration of the highly specific, high-affinity TP-TXA ${ }_{2}$ antagonist terutroban also resulted in prolonged survival after LLC resection in $40 \%$ of mice (Figure 3D). Conversely, the TP 
A

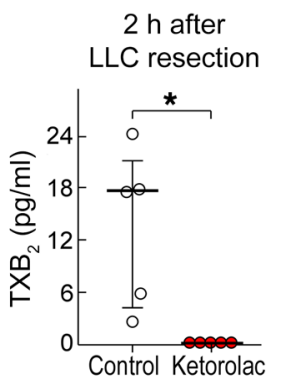

B

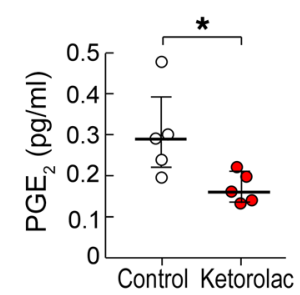

C

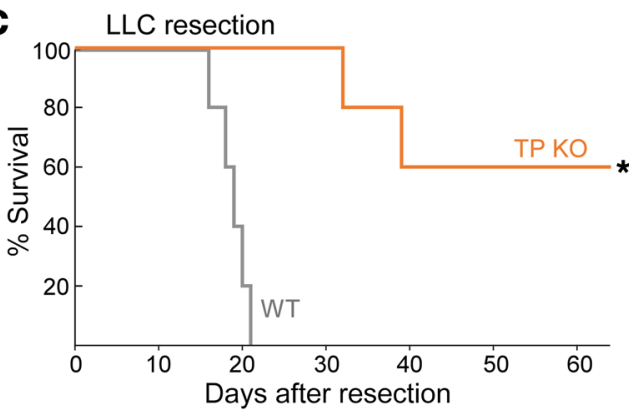

D

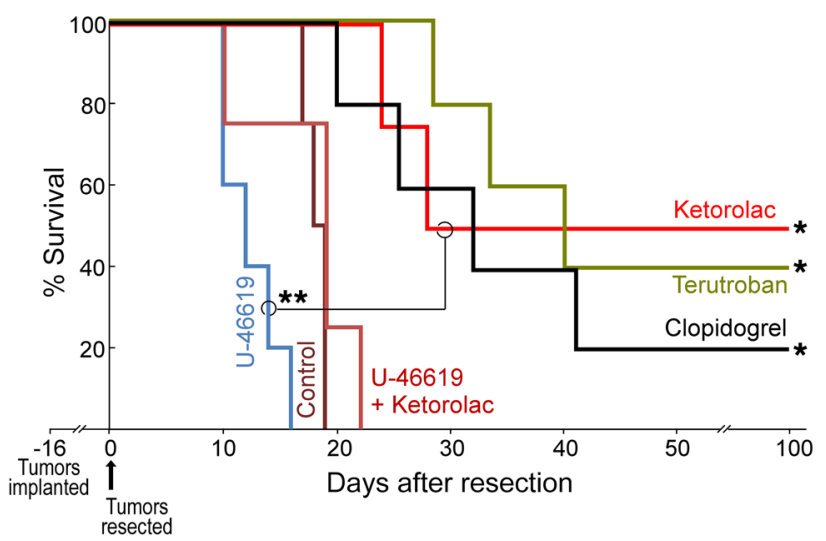

E
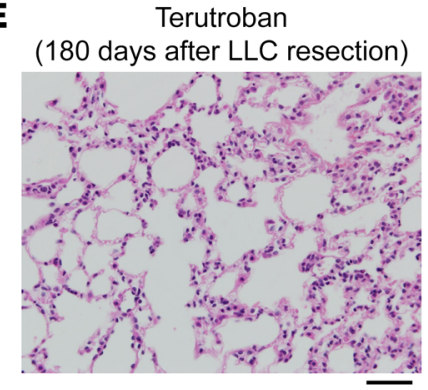

$\mathbf{F}$ (180 days after LLC resection)

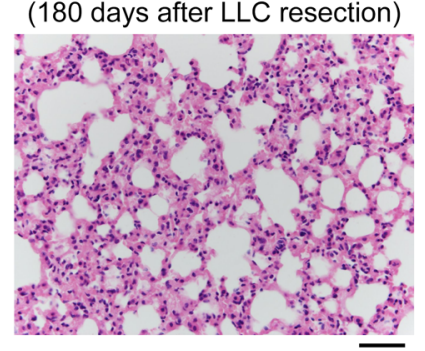

Figure 3. COX-1/TXA inhibition mediates the antitumor activity of ketorolac. LC-MS/MS-based analysis of (A) TXB or (B) PGE in plasma 2 hours after LLC resection from control or preoperative ketorolac-treated mice. $n=5$ mice/group. ${ }^{*} P<0.05$ vs. control. (C) Survival of TP KO mice vs. WT mice after LLC resection. $n=5$ mice/group. ${ }^{*} P=0.002$, WT vs. TP KO mice. (D) Preoperative clopidogrel, terutroban, U-46619, and/or ketorolac effects on survival after LLC resection. $n=4-5$ mice/group. ${ }^{*} P<0.01$, ketorolac, terutroban or clopidogrel vs. control. ${ }^{*} P=0.005 \mathrm{U}-46619$ vs. ketorolac. H\&E staining of lungs from preoperative (E) terutroban-, or (F) clopidogrel-treated mice at 180 days after LLC resection. Scale bars: $50 \mu \mathrm{m}$.

receptor agonist U-46619 accelerated morbidity from lung metastasis compared with control and abrogated the antitumor activity of ketorolac (Figure 3D). Since $\mathrm{TXA}_{2}$ stimulates platelet aggregation $(38,61)$, we also evaluated the activity of the antiplatelet agent clopidogrel. Preoperative clopidogrel prolonged survival after LLC resection compared with control, resulting in 20\% longterm survivors (Figure 3D). Similarly to preoperative ketorolac, no micrometastases were detected in the lungs of long-term survivors administered preoperative terutroban or clopidogrel on day 180 after LLC resection (Figure 3, E and F). Selective COX-2 inhibitors do not affect platelet $\mathrm{TXA}_{2}$ production and may not directly impair platelet aggregation $(38,60)$. Collectively, these results suggest that the antitumor activity of ketorolac is in part driven by COX-1/ $\mathrm{TXA}_{2}$ inhibition and subsequent reduction in platelet aggregation and accompanying degranulation.

Preoperative resolvins eradicate micrometastases and inhibit tumor-dormancy escape. LC-MS/MS analysis of plasma from preoperative ketorolac-treated mice after LLC resection demonstrated a global shift in the lipid-mediator profile (Supplemental Figure 2). Not only were COX-dependent and nonenzymatic oxidation products affected, but there was also shunting of the arachidonic acid cascade to the lipoxygenase (LOX) and cytochrome P450 (CYP) pathways. Specifically, ketorolac increased LOX-derived 12-oxoETE and 13-HODE as well as the tumorigenic CYP450-derived 5,6-EET, 11,12-EET, and 10,11-EDP compared with control (Sup- plemental Figure 2). This broad lipidomic shift indicates a complex balance between pro- and antitumorigenic mediators (53), which may account for the observed subset of ketorolac-treated mice that developed invasive lung metastasis (Figure 1A). Notably, lipoxin $\mathrm{A}_{4}\left(\mathrm{LXA}_{4}\right)$ was increased in the plasma of preoperative ketorolactreated mice after LLC resection (Figure $4 A$ ). Since $L_{X A}$ is an SPM (12), we reasoned that the balance of inflammation regulation may be tipped toward resolution, thereby either enhancing or recapitulating the antiinflammatory and proresolving activity of ketorolac. Indeed, SPMs, such as lipoxins and resolvins, possess potent antitumor activity in experimental models $(9,48-51,62)$.

To determine whether stimulation of inflammation resolution could prevent tumor recurrence and/or suppress outgrowth of micrometastases, we administered resolvins via osmotic pump 2 hours prior to tumor resection. Preoperative resolvin D2 (RvD2) prolonged survival after tumor resection and was more potent in preventing metastasis than RvD2 administered at the time of surgery (Figure 4B). Specifically, preoperative administration of resolvins ( $\mathrm{RvD} 2$, RvD3, or RvD4) resulted in 50\%-80\% survival at 37 days after resection, with RvD2 exhibiting the most potent antitumor activity (Figure 4C).

Since low-dose aspirin triggers the production of resolvins from omega-3 fatty acids (12), we treated mice with low-dose aspirin in combination with an omega-3 fatty acid diet and found increased survival, but no long-term survivors (Figure 4D). Although aspirin 
A

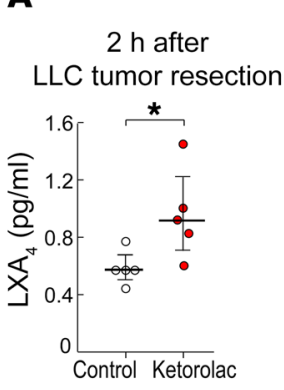

B LLC resection

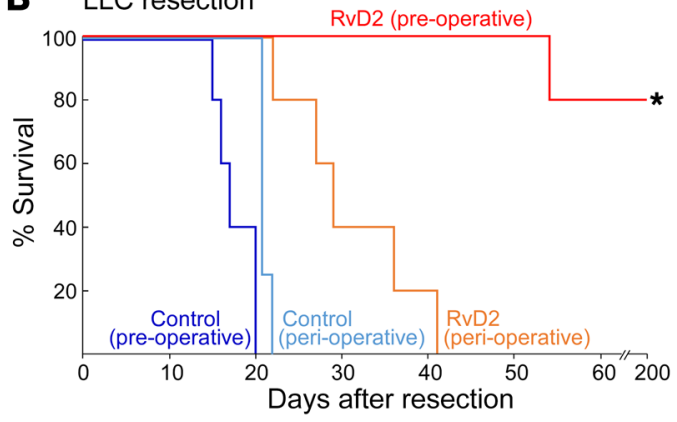

C

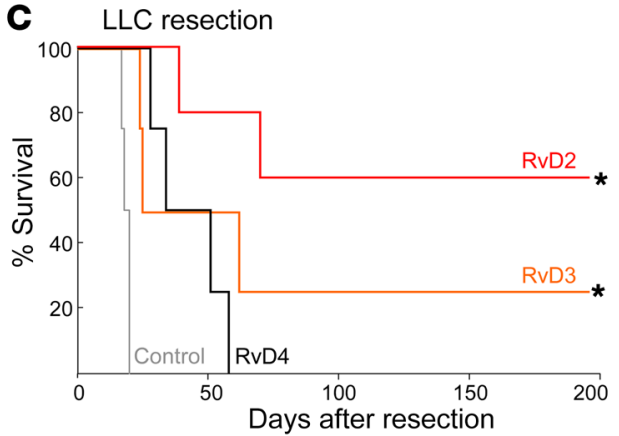

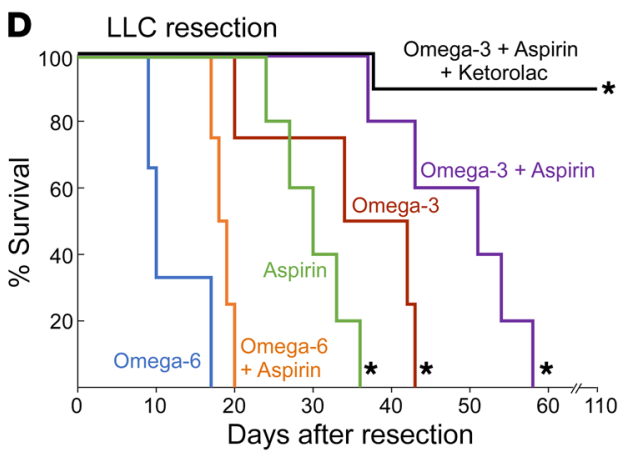

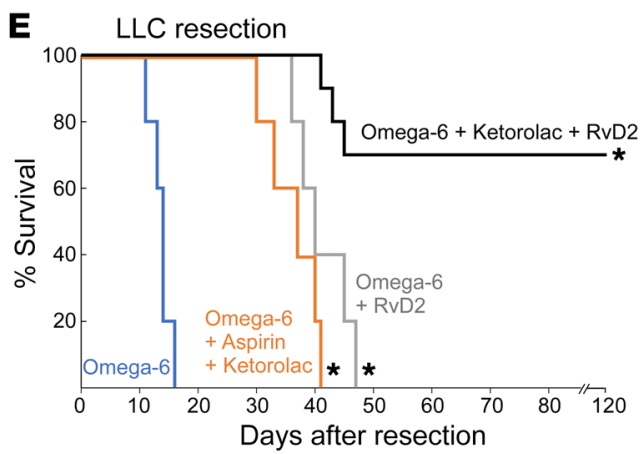

$\mathbf{F}$

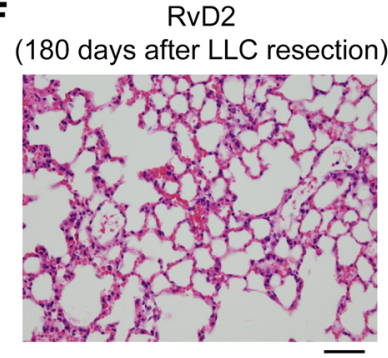

G Omega-3 + Aspirin + Ketorolac (180 days after LLC resection)

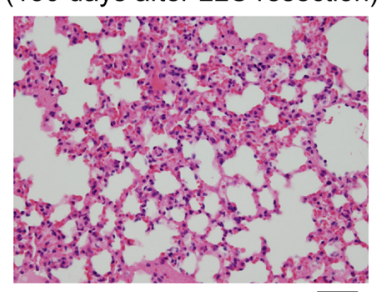

H

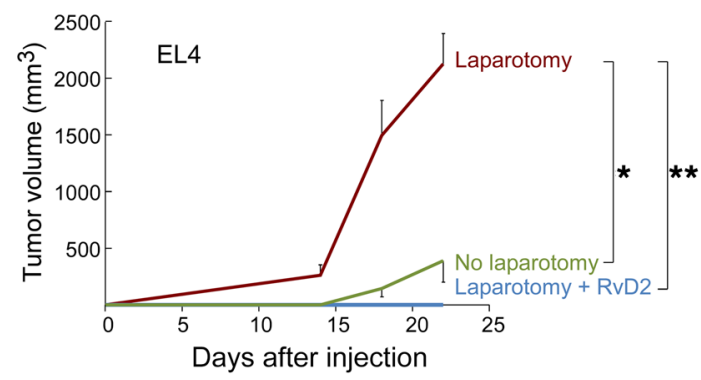

Figure 4. Preoperative resolvins eliminate micrometastases and prevent surgery-stimulated dormancy escape. (A) LC-MS/MS-based analysis of LXA in plasma 2 hours after LLC resection from control or preoperative ketorolac-treated mice. $n=5$ mice/group. ${ }^{*} P<0.05$ vs. control. (B) Preoperative vs. perioperative RvD2 effects on survival after LLC resection. $n=5$ mice/group. ${ }^{*} P<0.05$, preoperative RvD2 vs. perioperative RvD2. (C) Preoperative resolvins effects on survival after LLC resection. $n=5$ mice/group. ${ }^{*} P<0.05$, RvD2 or RvD3 vs. control. (D and E) Preoperative omega-3 or omega- 6 fatty acid diet and/or low-dose aspirin ( $10 \mathrm{mg} / \mathrm{kg} / \mathrm{d}$ ) effects on survival after LLC resection. Diet and aspirin were administered 7 days before and 3 days after LLC resection and preoperative ketorolac. $n=5$ mice/group. ${ }^{*} P<0.05$ vs. omega- 6 fatty acid diet. H\&E staining of lungs from mice treated with preoperative (F) RvD2 or (C) ketorolac, low-dose aspirin, and omega-3 fatty acid diet on day 180 after LLC resection. $n=4-5$ mice/group. Scale bars: $50 \mu \mathrm{m}$. (H) Growth of EL4 ( $10^{4}$ cells) after preoperative RvD2 and laparotomy. $n=5-10$ mice/group. ${ }^{*} P<0.05$, laparotomy vs. no laparotomy; ${ }^{* *} P<0.05$, laparotomy and RvD2 vs. laparotomy.

or omega-3 fatty acid diet alone exhibited less antitumor activity than the combination treatment, survival was prolonged compared with that of mice fed an omega- 6 fatty acid diet. The omega- 6 fatty acid diet alone accelerated progression and shortened median survival in mice from 19 days (control, Figure 2, A and B) to 16 days (Figure 4E) after resection, consistent with studies suggesting that dietary omega- 6 fatty acids can promote tumor progression and metastasis (53). Moreover, the omega- 6 fatty acid diet alone markedly shortened survival compared with combinations with aspirin, RvD2, and/or ketorolac (Figure 4E).

The potent antitumor activity of low-dose aspirin combined with omega-3 fatty acids offered the opportunity to examine whether promotion of resolution of inflammation could synergize with the antiinflammatory activity of ketorolac. Intriguingly, preoperative ketorolac in combination with low-dose aspirin and omega-3 fatty acid diet resulted in $90 \%$ survival at 110 days after resection (Figure $4 \mathrm{D})$. The combination of preoperative ketorolac and resolvins led to long-term survival after resection in $70 \%$ of mice administered the omega- 6 fatty acid diet (Figure 4E). Tumor cells were undetectable in H\&E-stained sections of lungs from long-term survivors treated with preoperative RvD2 or the combination of low-dose aspirin, omega-3 fatty acids, and ketorolac (day 180) (Figure 4, F and G).

To determine whether the antitumor activity of resolvins extended to a surgery-induced tumor-dormancy escape model, we injected $10^{4} \mathrm{EL} 4$ into mice and performed a laparotomy, which triggered dramatic tumor outgrowth. Preoperative RvD2 prevent- 
A
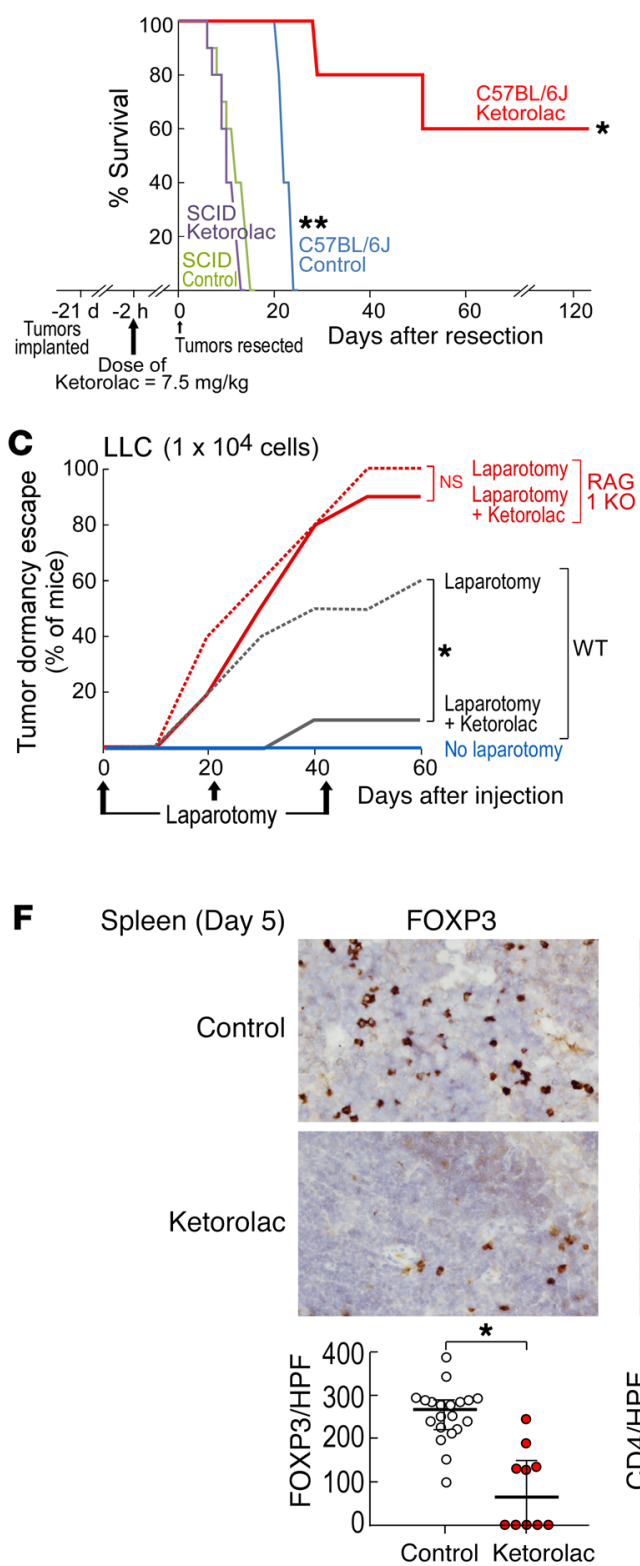

\section{$\mathbf{F}$}

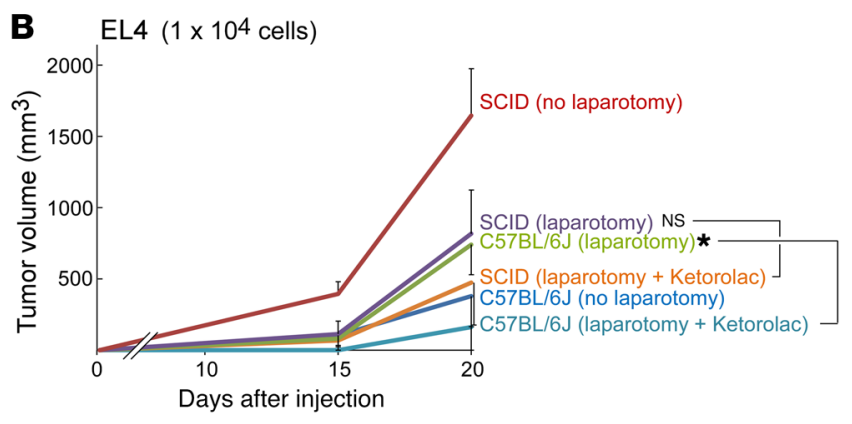

D

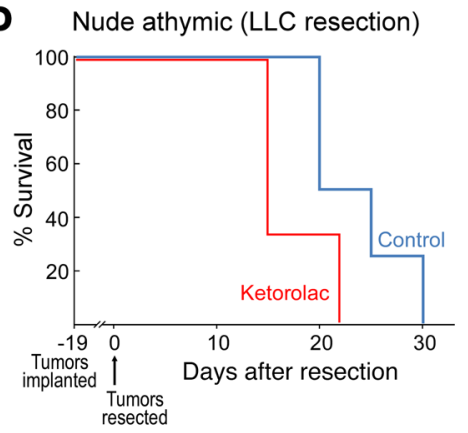

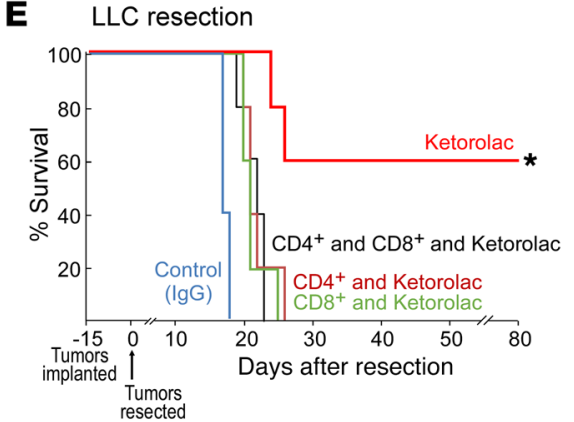
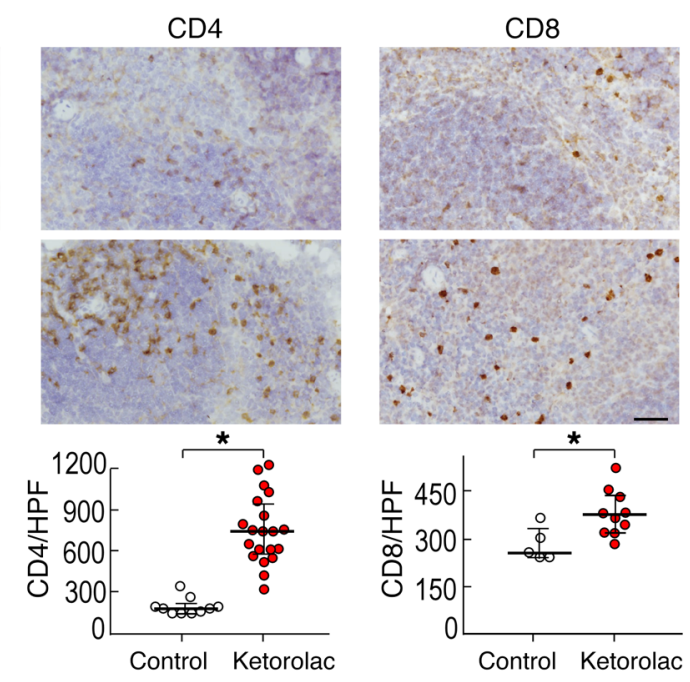

Figure 5. Ketorolac restores antitumor immunity. (A) Preoperative ketorolac effects on survival of SCID or [57BL/6) mice after LLC resection. $n=5-10$ mice/group. ${ }^{*} P=0.04,\left[57 \mathrm{BL} / 6\right.$ ) ketorolac vs. [57BL/6] control; ${ }^{*} P=0.004$, SCID control vs. C57BL/6) control (day 14 after resection). (B) Growth of EL4 ( $10^{4}$ cells) after preoperative ketorolac and/or laparotomy in SCID and C57BL/6) mice. Laparotomy was performed on day $0 . n=5-10$ mice/group. ${ }^{*} P<$ 0.05, C57BL/6) (laparotomy and ketorolac) vs. C57BL/6) (laparotomy). (C) Percentage of control or preoperative ketorolac-treated C57BL/6 or RAG1-KO mice demonstrating LLC tumor-dormancy escape. Laparotomies were performed on days 0,21 , and 42 after injection. $n=5-10$ mice/group. ${ }^{*} P<0.05$, WT (laparotomy and ketorolac) vs. WT (laparotomy). (D) Preoperative ketorolac effects on survival of athymic mice after LLC resection. $n=3-4$ mice/group. (E) Preoperative ketorolac and T cell depletion effects on survival after LLC resection. T cell-depleting (CD4+ or CD8+) antibodies or control (rat IgC2b) were administered 3 days before tumor resection, the day of tumor resection, and every 3 days thereafter for 2 weeks. $n=5$ mice/group. ${ }^{*} P<0.05$, control (IgC); $C D 4^{+}$and $C D 8^{+}$depletion and ketorolac; $C D 4^{+}$depletion and ketorolac or $C D 8^{+}$depletion and ketorolac vs. ketorolac. (F) Expression of FOXP3, CD4, and CD8 (brown DAB staining) in spleens from preoperative ketorolac-treated vs. control mice on day 5 after LLC resection. Scale bar: $100 \mu \mathrm{m}$. Immune cell quantification is represented as mean \pm SEM. $n=4-5$ mice/group. ${ }^{*} P<0.05$ vs. control.

ed surgery-induced EL4 tumor-dormancy escape (Figure $4 \mathrm{H})$. Thus, preoperative stimulation of inflammation resolution with resolvins may inhibit surgery-induced tumor recurrence.

The antitumor activity of ketorolac is $T$ cell dependent and impaired by chemotherapy. Inflammation plays a multifaceted role in regulating the adaptive immune response. For example, prostaglandins, such as $\mathrm{PGE}_{2}$, suppress acute inflammatory mediators, activate dendritic cells, sensitize $\mathrm{T}$ cells to eliminate infection, and promote $\mathrm{T}$ cell exhaustion $(36,42)$. Moreover, adaptive antitumor immunity has been implicated in the regulation of metastatic tumor dormancy (63, 64). These facts, along with the absence of tumor cells in the lungs of long-term survivors, led us to examine whether the immune system 
A

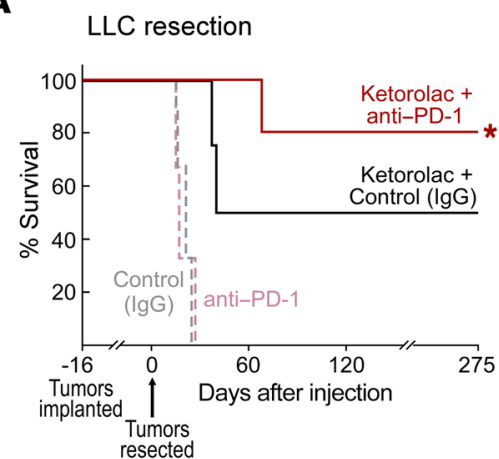

B

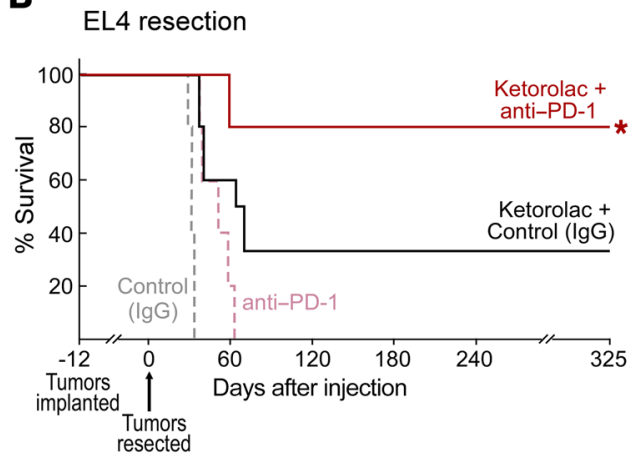

C

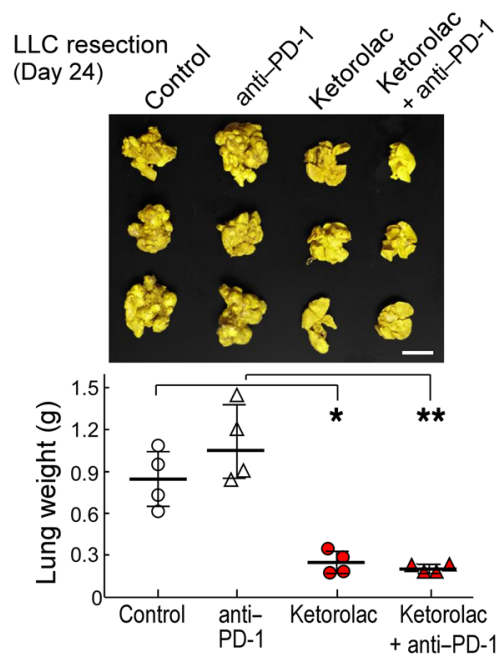

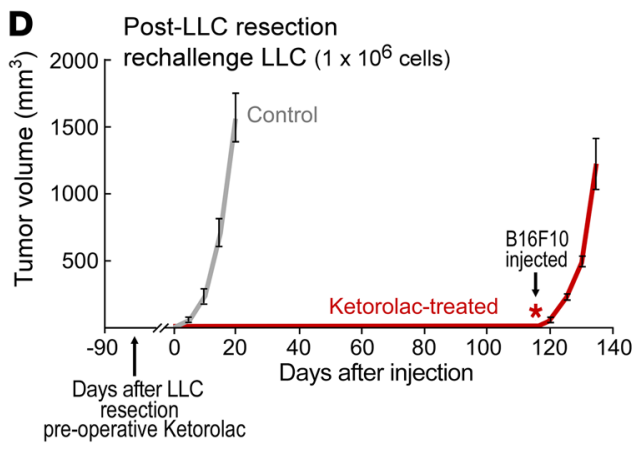
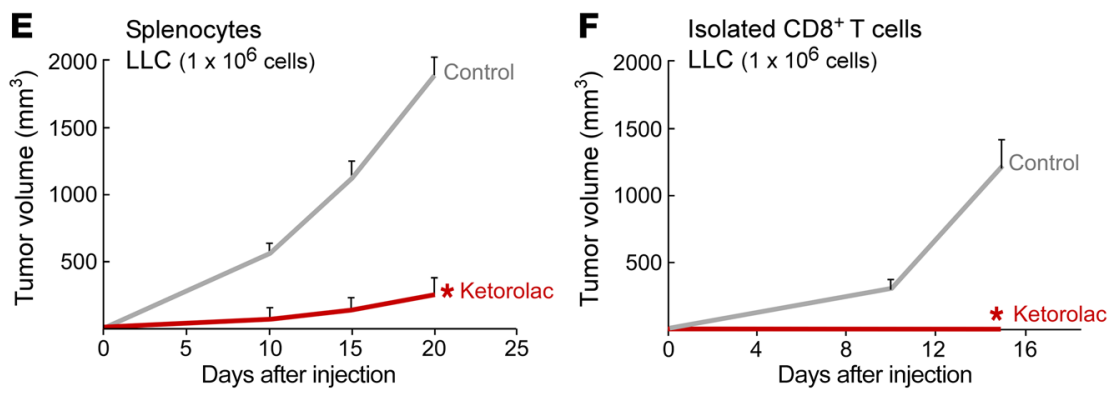

Figure 6. Antitumor activity of ketorolac is mediated by $\mathbf{T}$ cell immunity. (A and $\mathbf{B}$ ) Preoperative ketorolac and immune checkpoint blockade (anti-PD-1) on survival after tumor resection (LLC or EL4). Anti-PD-1 (200 $\mu \mathrm{g} /$ mouse $q 3$ days for 60 days). $n=5-10$ mice/group. $(\mathbf{A}) * P=0.002$, ketorolac and antiPD-1 vs. control (IgC) or anti-PD-1 alone. (B) ${ }^{*} P<0.05$, control (IgG), ketorolac and control (IgC), or anti-PD-1 vs. ketorolac and anti-PD-1. (C) Preoperative ketorolac and/or anti-PD-1 (1 dose, $200 \mu \mathrm{g} /$ mouse) effects on spontaneous LLC lung metastasis at 24 days after LLC resection. $n=4$ mice/group. Images show representative lung metastasis in preoperative ketorolac and/or anti-PD-1, and control mice. Scale bar: $1 \mathrm{~cm}$. Bar graph is presented as lung weight (g) \pm SEM per group. ${ }^{*} P=0.002$, ketorolac vs. control; ${ }^{* *} P<0.001$, ketorolac and anti-PD-1 vs. anti-PD-1. (D) Preoperative ketorolac-treated C57BL/6] mice that survived 90 days after LLC resection were injected with $10^{6} \mathrm{LLC}$ and compared with naive control mice. Ninety days later, ketorolac-treated mice were rechallenged with $10^{6}$ B16F10. ${ }^{*} P<0.05$, ketorolac vs. control. LLC tumor growth ( $10^{6}$ cells) after adoptive cell transfer of (E) splenocytes or (F) isolated $\mathrm{CD}^{+} \mathrm{T}$ cells from preoperative ketorolac-treated long-term survivors (90 days) after LLC resection or naive control mice. $n=3-5$ mice/group. (E and $\mathbf{F}$ ) ${ }^{*} P<0.05$, ketorolac vs. control.

plays a role in the antitumor activity of ketorolac. Preoperative ketorolac prolonged survival in $60 \%$ of immunocompetent C57BL/6J mice up to 120 days after LLC resection (Figure 5A). In contrast, ketorolac did not improve survival after resection in SCID mice, which lack functional B and T lymphocytes (Figure 5A). SCID mice exhibited a drastically shortened baseline survival in the LLC tumor-resection model, with a median overall survival of 10 days compared with 25 days in C57BL/6J mice (Figure 5A). Similarly, ketorolac did not affect laparotomy-induced tumor-dormancy escape in immunodeficient SCID or RAG1-KO mice (Figure 5, B and C).

To investigate the specific role of $\mathrm{T}$ cells in the antitumor activity of ketorolac, we utilized nude athymic mice in a C57BL/6J background. The antitumor activity of ketorolac was abrogated in these mice, as all of the ketorolac-treated mice succumbed to lung metastasis by day 22 after LLC resection (Figure 5D). To confirm that the observed antitumor activity of ketorolac was $\mathrm{T}$ cell mediated, we depleted $\mathrm{CD}^{+}$and/or $\mathrm{CD} 4^{+} \mathrm{T}$ cells in vivo using neutralizing antibodies. $\mathrm{T}$ cell depletion of either subtype abolished the antitumor activity of ketorolac (Figure 5E), confirming that both
T cell subtypes are necessary for the ketorolac-induced antitumor response. Immunohistochemistry revealed increased $\mathrm{CD}^{+}$and $\mathrm{CD}^{+} \mathrm{T}$ lymphocytes and decreased cells positive for the regulatory $\mathrm{T}$ cell marker FOXP3 in the spleen and lung tissues of ketorolac-treated mice on days 5 and 7 after resection, respectively, as compared with control mice (Figure 5F and Supplemental Figure $3 \mathrm{~A})$. RvD2 also increased CD8 ${ }^{+} \mathrm{T}$ lymphocytes in the lung tissues of mice (day 180 after resection) (Supplemental Figure 3B). Therefore, the antitumor activity of ketorolac and resolvins may be mediated by activation of $\mathrm{CD}^{+}$and/or $\mathrm{CD} 8^{+} \mathrm{T}$ cells.

Since checkpoint inhibitors such as anti-programmed cell death protein 1 (anti-PD1) antibody can augment cytolytic $\mathrm{T}$ cell responses, we hypothesized that preoperative ketorolac and immune-checkpoint blockade may act synergistically in our resection models. Indeed, this combination resulted in synergistic antitumor activity, with approximately $80 \%$ of animals exhibiting long-term survival in both syngeneic LLC and EL4 tumor-resection models (Figure 6, A and B). While ketorolac alone exhibited antitumor activity that was further enhanced by anti-PD1 treat- 


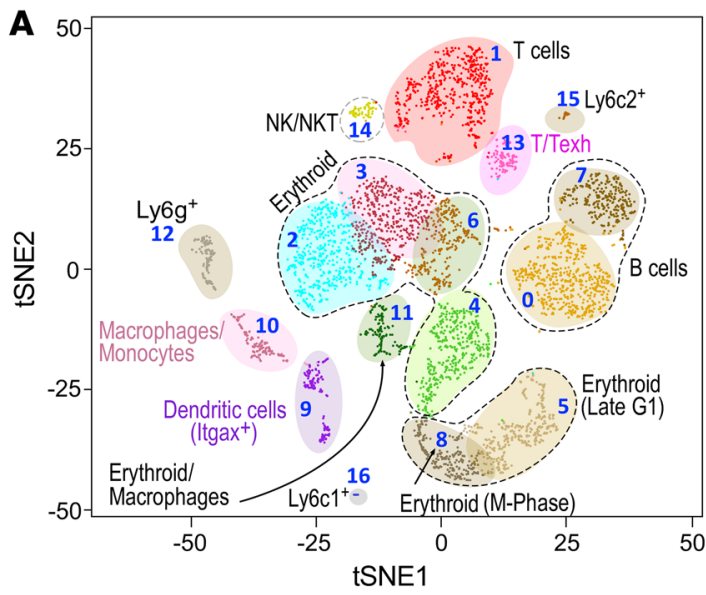

C

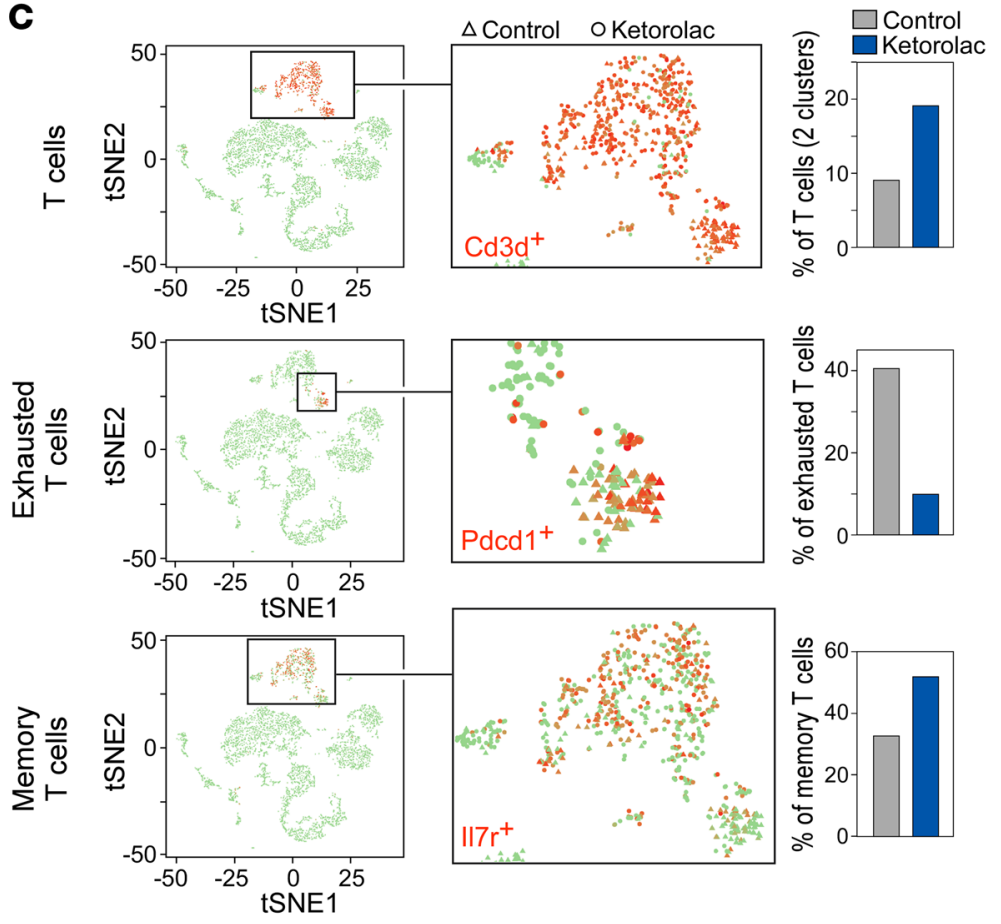

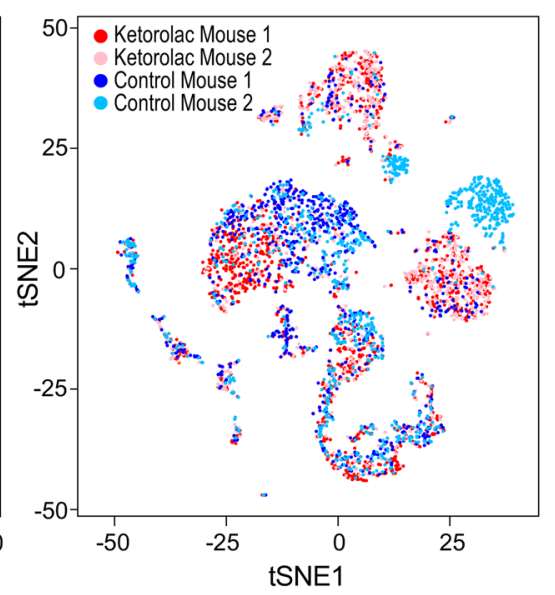
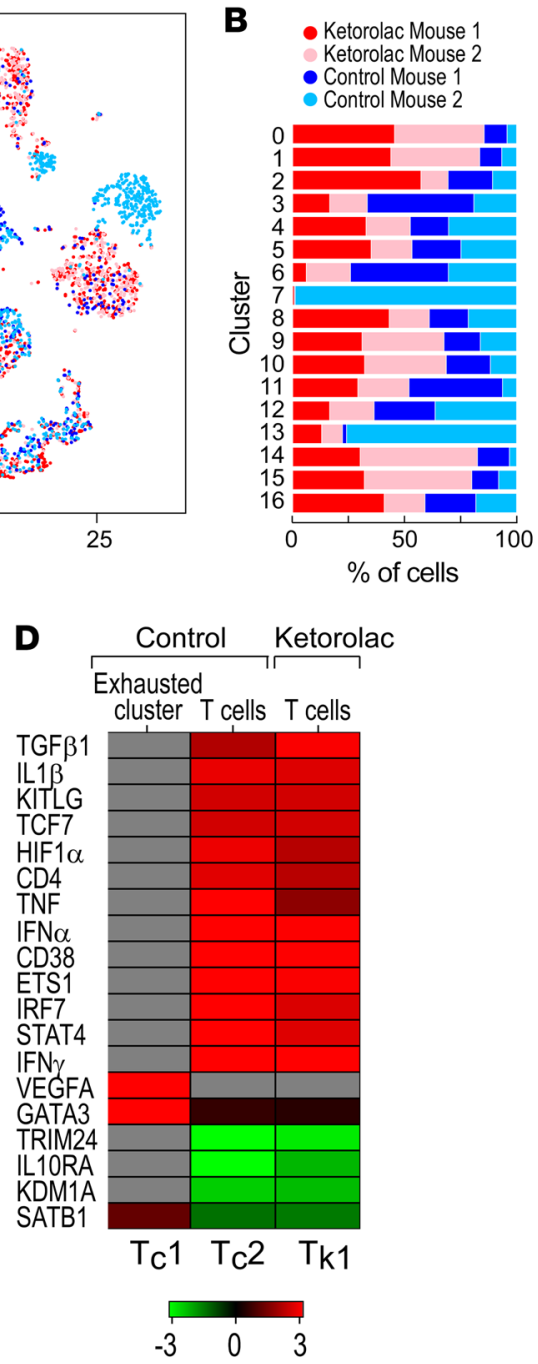

Figure 7. ScRNA-Seq analysis demonstrates an altered immune landscape in response to ketorolac. ScRNA-Seq analysis on splenic cells isolated from preoperative ketorolac-treated or control mice 7 days after LLC resection. $n=2$ mice/group throughout. (A) 2D visualization of single-cell clusters generated using the tSNE approach from normalized data of 2429 ketorolac-treated and 1864 control splenic cells. Cell clusters were annotated based on expression of established immune cell markers, e.g., T cells (Cd3d+), exhausted T cells (Pdcd1, Ctla4, Lag3, Entpd1, and TIM3), T memory cells (II7r, Ccr7, Sell, CD44), B cells $\left(C d 19^{+}\right)$, dendritic cells (Itgax), and macrophages (Adgre1) (left panel). Chart depicts relative proportions of cells in the clusters from each sample (right panel). (B) Percentage of cells from control and ketorolac-treated mice, per cluster. (C) Preoperative ketorolac effects on the immune landscape of T cells. Feature maps depict the expression of T cell (Cd3d+), exhausted T cell (Pdcd1), and memory T cell (II7r) markers for each cell cluster on the tSNE map. Cells from ketorolac-treated and control samples are shown as solid dots and triangles, respectively. Bar plots depict the relative proportions of T cell subpopulations in control and ketorolac-treated single-cell profiles. (D) Heatmap depicts the activation or inhibition of key regulators in the control and ketorolac-treated T cell clusters. Regulators were calculated based on $Z$ scores using an upstream regulator analysis module in ingenuity pathways analysis systems.

ment, anti-PD1 alone did not exhibit antitumor activity in these models (Figure 6, A-C). Moreover, preoperative ketorolac alone or in combination with anti-PD1 suppressed lung metastasis on day 24 after LLC resection (Figure 6C).

To corroborate the antitumor immunity stimulated by ketorolac, we assessed immunological memory by rechallenging long-term survivors administered ketorolac prior to tumor resection with the identical tumor type ( $10^{6} \mathrm{LLC}$ cells). These long-term survivors were resistant to LLC rechallenge, as no visible tumors were observed over
100 days after injection. When these mice were injected with a second syngeneic tumor type $\left(10^{6} \mathrm{~B} 16 \mathrm{~F} 10\right)$, they exhibited rapid tumor growth (Figure 6D), suggesting tumor-specific immunity. Similarly, preoperative ketorolac-treated long-term survivors in the postlaparotomy and postchemotherapy LLC tumor-dormancy escape models were resistant to LLC challenge, but exhibited rapid tumor growth when challenged with B16F10 tumor cells (Supplemental Figure 4, A and B). Moreover, preoperative RvD2-treated long-term survivors after LLC resection were also resistant to LLC rechallenge for over 
A Granulocytes

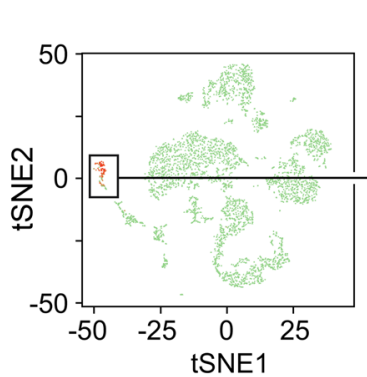

$\Delta$ Control

O Ketorolac
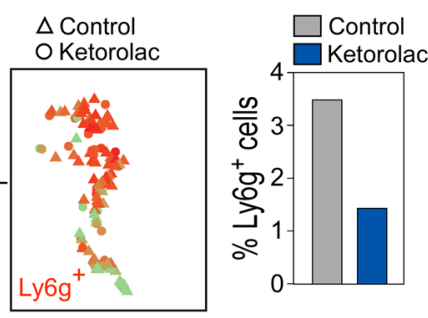

tSNE 1
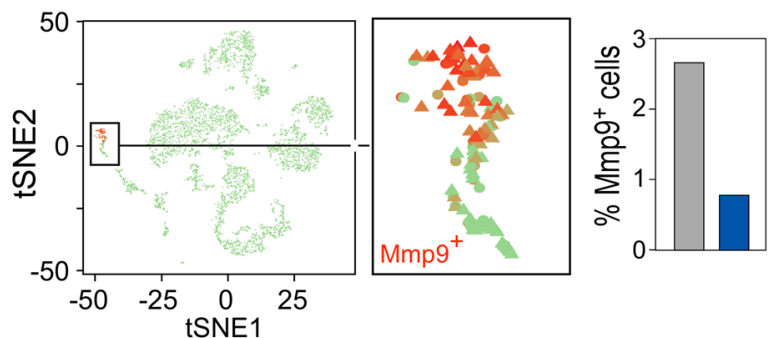

Figure 8. Key regulators in granulocytes/neutrophils from preoperative ketorolac-treated and control mice. Spleens from preoperative ketorolac-treated and control mice are identical to those used in Figure 7. (A) Feature and bar plots highlight the proportion of Ly6g'- and Mmp9-producing granulocytes/neutrophils in spleens from ketorolac-treated or control mice. (B) Interactive network of key regulators significantly activated in granulocytes/neutrophils from control mice. Bar graph depicts $Z$ score ( $>1.5$ = activation, $<1.5$ = inhibition) of NF- $\kappa B$ and $\mathrm{PI} 3 \mathrm{~K}$ complexes in control and ketorolac-treated samples based on expression of genes in the granulocyte/neutrophil cluster.

90 days after injection, yet developed aggressive tumor growth when injected with B16F10 (Supplemental Figure 4C). To confirm that the antitumor activity of ketorolac may be mediated by $\mathrm{CD} 8^{+} \mathrm{T}$ cell activation, we performed adoptive transfer of immune cells from preoperative ketorolac-treated long-term survivors after LLC resection into naive mice. Splenocyte or $\mathrm{CD}^{+} \mathrm{T}$ cell transfer from long-term ketorolac-treated survivors inhibited LLC growth compared with that of control mice that received splenocytes or $\mathrm{CD} 8^{+} \mathrm{T}$ cells from naive mice (Figure 6, E and F, and Supplemental Figure 5, A-D).

Cancer patients undergoing tumor resection often receive adjuvant chemotherapy, as perioperative chemotherapy may be most effective when administered before surgery, at the time of surgery or soon after surgery $(65,66)$. Thus, we determined whether administration of chemotherapy after preoperative ketorolac would affect survival and antitumor immunity after tumor resection. A single dose of cisplatin on day 7 after LLC resection abrogated the long-term survival observed in the preoperative ketorolac group (Supplemental Figure 6A). Immunohistochemistry revealed that chemotherapy following preoperative ketorolac reduced the number of $\mathrm{CD}^{+}$and $\mathrm{CD} 8^{+} \mathrm{T}$ cells compared with preoperative ketorolac alone on day 7 after LLC resection (Supplemental Figure 6B). Adjuvant chemotherapy is typically administered 4 to 6 weeks after surgery. We therefore asked whether delayed adminis-

B

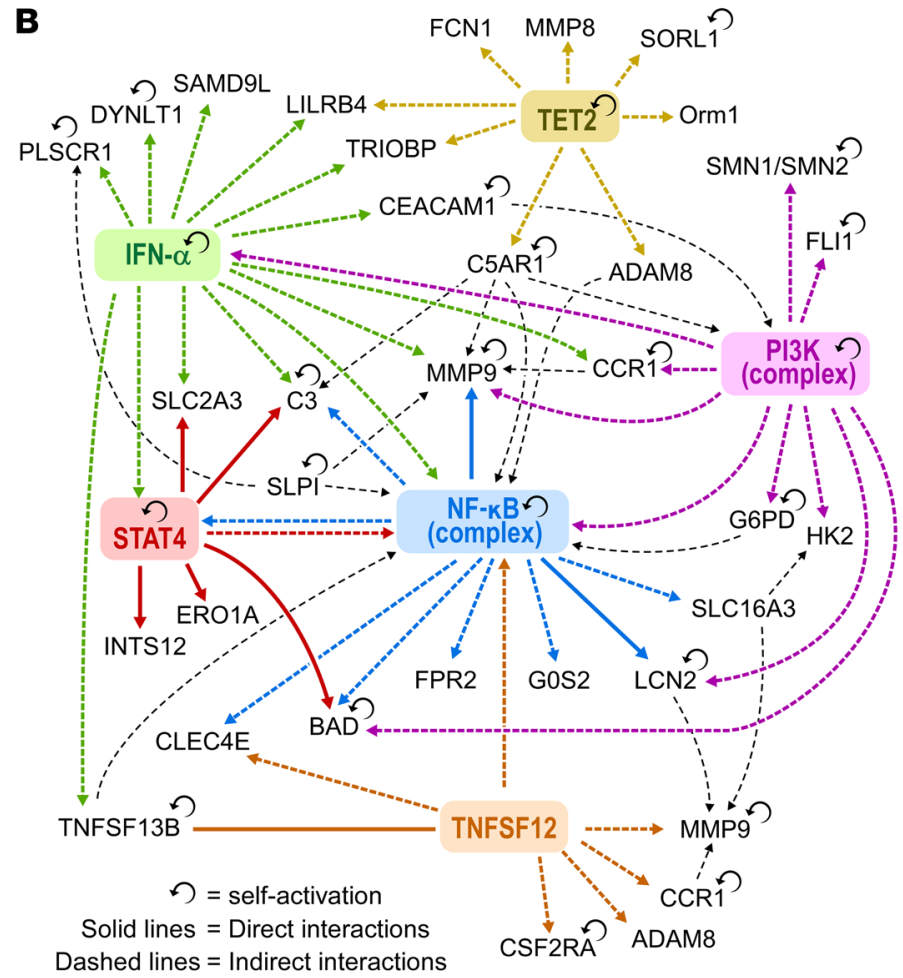

Dashed lines $=$ Indirect interactions
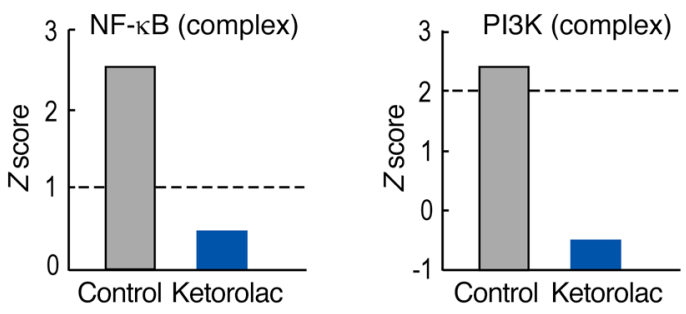

tration of chemotherapy would also influence preoperative ketorolac-treated long-term survivors. Ketorolac-treated mice surviving over 90 days after LLC resection were subjected to 3 cycles of cisplatin ( $5 \mathrm{mg} / \mathrm{kg} \mathrm{q} 5$ days) and then rechallenged with LLC tumor cells. This challenge resulted in a rapid onset of LLC tumor growth in the ketorolac-treated as well as control mice (Supplemental Figure 6C). Therefore, adjuvant chemotherapy, even when administered a few weeks after tumor resection, may interfere with the antitumor immunity of preoperative ketorolac.

Single-cell profiling of immune cell populations in response to ketorolac. To characterize the ketorolac-mediated immunomodulatory response, we utilized droplet-based single-cell RNA-Seq (scRNA-Seq) to obtain single-cell resolution transcriptomes of splenic tissues from control and ketorolac-treated mice on day 7 after LLC resection. The transcriptome profiles of 1864 and 2429 individual cells from spleens of 2 control and 2 ketorolac-treated mice, respectively, revealed that ketorolac modulated the immune landscape by altering the relative abundance and transcriptome profiles of T cells and granulocytes. Standard cluster analysis of cells based on their most variably expressed genes in the scRNASeq transcriptomes is shown in the projection of gene expression space onto a single 2D space using t-distributed stochastic neighbor embedding (tSNE) (Figure 7A). The 2D plot contains cells 
from both ketorolac-treated and control samples analyzed as 1 concatenated data set. Analysis of marker genes assigned the 17 identified robust clusters to T cells, B cells, erythroid cells, NK cells, granulocytes, neutrophils, macrophages, and dendritic cells (Figure 7A). Only 1 cell cluster was mouse specific and unrelated to treatment, while the majority of clusters were formed by all mice and represent known cell types - indicating minimal batch or mouse effect (Figure 7A). Ketorolac-treated spleens differed from control in relative cell-type abundance and gene expression states of individual cells.

At the level of cell-type abundance, with the exception of a few clusters that contained only or predominantly cells from control samples, the clusters contained varying proportions from both control and ketorolac-treated mice (Figure 7B). In both mice, ketorolac increased the fraction of splenic $\mathrm{Cd} 3 \mathrm{~d}^{+} \mathrm{T}$ cells compared with those of control, from $9.12 \%$ to $19.1 \%$ (clusters 1 and 13 in Figure 7A). One $\mathrm{T}$ cell cluster (cluster 13 in Figure 7A) was enriched in exhausted $\mathrm{T}$ cells (as determined by expression of markers PD1, CTLA4, and TIM3) from 1 control mouse, while both ketorolac-treated mice had low numbers of exhausted T cells (Figure 7, B and C). Moreover, the proportion of memory T cells (IL7r+) (cluster 1 in Figure 7A) increased from 32\% in the 2 control spleens to $52 \%$ in the 2 ketorolac-treated spleens (Figure 7C). Although T cell-subtype abundance varies considerably between individuals (67), this finding is consistent with systemic activation of $\mathrm{T}$ cell immunity in the ketorolac-treated mice. This constellation suggests that ketorolac may improve $\mathrm{T}$ cell antitumor immunity and the presence of exhausted PD1 ${ }^{+} \mathrm{T}$ cells is consistent with ketorolac's synergism with an anti-PD1 inhibitor (Figure 6, A and B).

At the transcriptome level within the $\mathrm{T}$ cell clusters, shifts in cell phenotypic states of control and ketorolac-treated mice (enrichment of cells from 1 mouse in a subregion of a cluster, Figure 7A) are apparent in the tSNE plots. Utilizing a systematic quantitative analysis, we focused on $\mathrm{T}$ cells and identified genes that were significantly enriched in each cluster $(P<0.01)$ and then performed a pathway analysis on these genes for the control and ketorolac-treated mice independently. Such gene ontology analysis suggested that distinct upstream regulators are significantly activated or inhibited in the different $\mathrm{T}$ cell clusters from control and ketorolac-treated mice (Figure 7D). The upstream regulator analysis (68) on genes associated with the exhausted $\mathrm{T}$ cell cluster in the control spleens indicated states controlled by transcription factor GATA3 and the cytokine VEGFA, which are associated with angiogenesis and Th2 polarization (69). In contrast, the same analysis in spleen cells from ketorolac-treated mice suggested activation of a state under control of the transcription factor 7 (TCF7) and the cytokines TNF- $\alpha$, IFN- $\gamma$, and IFN- $\alpha$ (Figure 7D), which are associated with activated cytotoxic T cells. Of note, TCF7, which is associated with memory $\mathrm{T}$ cells and implicated in rescuing $\mathrm{T}$ cells from exhaustion by anti-PD-1 therapy (70), was activated in the $\mathrm{CD}^{+} \mathrm{T}$ cells of ketorolac-treated mice.

In addition, scRNA-Seq analysis demonstrated reduced $\mathrm{Ly}_{6 \mathrm{~g}} \mathrm{~g}^{+}$ granulocytes in spleens of both ketorolac-treated mice (35 out of 2429 cells, $1.4 \%$ ) as compared with the control mice (65 out of 1864 cells, 3.5\%) (Figure 8A, cluster 12 in Figure 7A). A significant global shift in gene expression profiles of granulocytes from ketorolactreated versus control mice was not observed. However, the pro- portion of Mmp9-expressing Ly6g $\mathrm{g}^{+}$cells was significantly higher in the spleens of control compared with ketorolac-treated mice (Figure 8A). MMP9 is a critical protein that promotes the expansion of

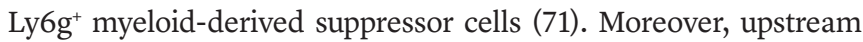
regulator analysis (68) of the granulocyte cluster indicated that $\mathrm{NF}-\kappa \mathrm{B}$ and PI3K were significantly activated in granulocytes from control mice compared with ketorolac-treated mice (Figure 8B), which is consistent with the suppressive activity of NSAIDs (72). The pathways and functions enrichment analysis also depicted alterations in triacylglycerol, carbohydrate, and lipid metabolism in ketorolac-treated granulocytes (Supplemental Table 1). We speculate that the low PI3K activity in the ketorolac-treated samples may decrease glycolysis (73), the major pathway of energy generation for granulocytes (74), and diminish migration (75).

\section{Discussion}

Here, we demonstrate that preoperative, but not postoperative, ketorolac and/or resolvins eradicate micrometastases in multiple tumor-resection models. While we cannot exclude minimal residual disease due to individual disseminated malignant cells, the elimination of metastatic recurrence by preoperative ketorolac resulted in long-term survival after tumor resection. These interventions also prevented dormancy escape induced by surgery or chemotherapy in several tumor types. The antitumor activity of ketorolac is dependent on a T cell-mediated response, as its activity was enhanced by immune checkpoint blockade and abrogated in athymic mice. Consistent with the immunohistochemistry, single-cell transcriptomics demonstrated increased $\mathrm{CD}^{+}$and $\mathrm{CD}^{+} \mathrm{T}$ cells in the spleens of ketorolac-treated mice as well as a shift in the proportions of $\mathrm{T}$ cell subtypes, indicating an increase in memory $\mathrm{T}$ cells and reduction of exhausted $\mathrm{T}$ cells. The $\mathrm{T}$ cell response may be specific for tumor cells, since ketorolac-treated mice were protected against a secondary challenge with the same tumor type, but not from an unrelated type. Moreover, adoptive transfer confirmed that the protective antitumor activity of ketorolac was $\mathrm{T}$ cell mediated, as no tumor growth was observed in mice that received $\mathrm{T}$ cells from ketorolac-treated long-term survivors. Therefore, targeting cancer therapy-induced inflammation with preoperative ketorolac and/or resolvins may reduce tumor recurrence and metastatic outgrowth via a $\mathrm{T}$ cell response.

Our study provides insight into the multifaceted roles of cyclooxygenase enzymes and their products in tumor biology. Specifically, we establish the importance of inhibiting COX-1 while maintaining COX-2 activity to control tumor recurrence or metastasis. These results shed light on the conceptual framework of inflammation and adaptive immunity in cancer. For instance, COX inhibition is generally thought to contribute to the prevention and control of tumor growth $(38,76)$. In the context of surgery, the NSAID meloxicam was recently reported to suppress dormancy escape of micrometastatic lesions in mouse models (2). However, we show that COX-2 inhibition may hinder the antitumor activity of ketorolac via impairment of the resolution of inflammation. In fact, selective COX-2 inhibitors have not improved recurrence-free survival in the clinic (77-81). Rather, chronic COX-2 inhibition or NSAIDs (e.g., sulindac) are linked to increased risk of breast and hematological cancers and can result in resistant adenomas and breakthrough carcinomas $(82,83)$. 
Moreover, while a combination of COX-2 inhibitors with adjuvant temozolomide following resection of glioblastoma extended progression-free survival, the COX-2 inhibitors also increased the rate of metastasis (84).

In addition, COX-2-derived $\mathrm{PGE}_{2}$ exhibits complex and opposing activities. $\mathrm{PGE}_{2}$ has been implicated in the suppression of antitumor immunity (85-87), as it can hinder antigen presentation, inhibit $\mathrm{T}$ cell and $\mathrm{NK}$ cell effector functions, and promote the activity of regulatory $\mathrm{T}$ cells $(36,85,88)$. In contrast, $\mathrm{PGE}_{2}$ may be necessary for optimal $\mathrm{CD}^{+} \mathrm{T}$ cell response $(38,89)$, supporting our finding that baseline COX-2 is critical for the antitumor immune response of ketorolac. $\mathrm{PGE}_{2}$ also plays a critical role in the termination of inflammation, as it is required for a bioactive lipid-class switching to generate SPMs that promote the resolution of inflammation (40). The proresolution activities of $\mathrm{PGE}_{2}$ on both the innate and adaptive immune system (12) may explain why selective COX-2 inhibition with celecoxib impaired the antitumor activity of preoperative ketorolac (Figure 2C).

The importance of COX-1 inhibition in cancer is poorly characterized and has not been translated to cancer patients (38). TXA synthesis is independent of COX-2 (90), which is consistent with our finding that the antitumor activity of preoperative ketorolac is mediated by COX-1/TXA 2 inhibition. In addition, $20 \%$ of mice treated preoperatively with the antiplatelet agent clopidogrel demonstrated long-term survival in our tumor-resection model, consistent with reports showing that clopidogrel prevents carcinogenesis by restoring antitumor immunity, including increasing the number of tissue $\mathrm{CD}^{+} \mathrm{T}$ cells (91). Clopidogrel also stimulates $\mathrm{CD}^{+}$and $\mathrm{CD} 25^{+} \mathrm{T}$ cells in an atherosclerosis model (92). Studies suggest a direct role for $\mathrm{TXA}_{2}$ in dendritic cell maturation and neutrophil migration (90), which may account for our genetic and pharmacologic data on the role of the $\mathrm{TXA}_{2}$ receptor in reducing postresection tumor recurrence.

Our findings have important therapeutic implications. First, ketorolac may increase long-term survival in multiple cancer types if administered preoperatively, rather than postoperatively, per current standards of care. Second, while ketorolac is a simple, inexpensive, and relatively nontoxic treatment, potential concerns include the risk of bleeding, wound dehiscence, and acute kidney injury. These side effects were not noted in a human retrospective study (34). In contrast, SPMs dampen inflammatory mediator release from platelets and do not appear to increase the risk of bleeding nor to enhance the hemostatic function of platelets $(12,93)$. In our animal studies, ketorolac and resolvins demonstrated synergistic antitumor activity without overt toxicity. Third, our results suggest that coadministration of preoperative ketorolac and immune checkpoint inhibitors may provide optimal therapeutic efficacy, consistent with recent studies (2, 24, 25). High-risk stage III melanoma may be an ideal setting to assess the potential therapeutic benefit of combining ketorolac and checkpoint inhibitors, as checkpoint inhibitors alone have been shown to decrease risk of recurrence (94). Fourth, although adjuvant chemotherapy increases overall survival in select cancer patients, it may also harbor inherent tumor-promoting activity $(9,10,18,95-97)$ limiting its overall efficacy, including inflammation-associated impairment of antitumor immunity that could stimulate micrometastatic disease. Indeed, administration of chemotherapy within 7 days of surgery was detrimental to achieving effective antitumor $\mathrm{T}$ cell immunity, which may be due to cytotoxicity on proliferating $\mathrm{T}$ cells. Even when administered several weeks after resection, chemotherapy appears to abrogate $\mathrm{T}$ cell memory and may increase the likelihood of recurrence. Fifth, dormant tumor cells are typically resistant to adjuvant chemotherapy (98), and chemotherapy can awaken dormant micrometastases. Although we did not specifically study the combination of resolvins and ketorolac with chemotherapy, tumor-dormancy escape was inhibited in a subset of mice when ketorolac was administered alongside chemotherapy (Figure 1, F-H). We also found via LC-MS/MSbased profiling that ketorolac stimulated an increase in plasma $\mathrm{LXA}_{4}$. Moreover, systemic treatment with resolvins inhibits chemotherapy-stimulated tumor growth in multiple tumor models (9). Collectively, our data suggest that flanking chemotherapy with antiinflammatory or proresolution therapies may be a novel approach for cancer treatment. Sixth, cancer patients in remission may benefit from preoperative ketorolac or resolvins prior to surgery, since surgery or even biopsy can induce tumordormancy escape or metastasis $(1,6,8)$. The relapse pattern following delayed reconstructive surgery in breast cancer patients is bimodal, suggesting a systemic action on dormant micrometastases from surgery (30). Seventh, although it may be tempting to enroll patients at highest risk for recurrence in trials of ketorolac or resolvins, these patients are likely to receive neoadjuvant or adjuvant chemotherapy, radiation (encompassing draining lymph nodes), and/or have their draining lymph nodes resected per current clinical guidelines. These interventions may counteract the induction of an effective $\mathrm{T}$ cell immune response. It may therefore be prudent to first conduct small biomarker endpoint studies to assess the impact of preoperative ketorolac and/or resolvins on $\mathrm{T}$ cell response via monitoring activation of $\mathrm{CD}^{+}$cells after surgery, $\mathrm{T}$ cell response to predicted neoantigens, T cell receptor diversity, or single-cell sequencing. Thus, if neoadjuvant chemotherapy negates an antitumor $\mathrm{T}$ cell response despite administration of preoperative ketorolac, such patients would then be excluded from a randomized prospective study.

Collectively, our data suggest a paradigm shift in clinical approaches to resectable cancers. Over the last century, the field has evolved from a "more is better" Halstedian stance with increasingly radical surgeries to a view of early stage cancer as a systemic disease, resulting in the adoption of adjuvant and neoadjuvant chemotherapy. Now, we and others (2) are showing that it may be possible to eradicate micrometastatic disease and dormant tumor cells without chemotherapy. Here, we demonstrate that unleashing $\mathrm{T}$ cell immunity by preoperative suppression of systemic inflammation or stimulation of inflammation resolution exhibits potent antitumor activity, even curing mice of micrometastases. Pharmacological enhancement of resolution via exogenous resolvins (demonstrated here with RvD2, RvD3, and $\mathrm{RvD} 4$ ) at ng/d doses or omega-3 fatty acid supplementation restores endogenous SPMs. Moreover, ketorolac and resolvins demonstrate synergistic antitumor activity. Thus, simultaneously blocking proinflammatory responses with ketorolac and activating endogenous resolution programs via resolvins may represent a novel approach for preventing systemic recurrence in the context of locoregional disease. 


\section{Methods}

Tumor resection. Tumors were resected at $1500-2000 \mathrm{~mm}^{3} .10^{6} \mathrm{LLC}$ cells (ATCC) were subcutaneously injected into male C57BL/6J, SCID, athymic C57BL/6J, TP KO, WT, or COX-2-KO mice (Jackson Laboratory). Experiments were repeated 3 times with similar results. For orthotopic tumors, $5 \times 10^{4} 4 \mathrm{~T} 1$ (ATCC) or E0771 ( $\mathrm{CH} 3$ BioSystems) were injected into the fourth mammary fat pad of female BALB/cJ or C57BL/6J mice, respectively.

Drug administration. Drugs were administered preoperatively (2 hours before surgery), at the time of surgery, or postoperatively (2 hours after surgery). For chemotherapy, mice were treated with preoperative ketorolac $(7.5 \mathrm{mg} / \mathrm{kg})$ and a postoperative dose of cisplatin $(5 \mathrm{mg} / \mathrm{kg})$ on day 7 after tumor resection. For immune-checkpoint blockade, mice were treated preoperatively with anti-mouse PD-1 (CD279; clone RMP1-14) (200 $\mu \mathrm{g} / \mathrm{mouse}$; Bio X Cell). For COX inhibition, mice were treated preoperatively with FR122047 (20 mg/ $\mathrm{kg})$, TFAP (30 mg/kg), SC-560 (30 mg/kg), celecoxib (60 mg/kg), indomethacin $(3 \mathrm{mg} / \mathrm{kg})$, ibuprofen $(30 \mathrm{mg} / \mathrm{kg})$, diclofenac $(10 \mathrm{mg} /$ $\mathrm{kg}$ ), or aspirin (10 or $30 \mathrm{mg} / \mathrm{kg}$ ). For $\mathrm{TXA}_{2}$ inhibition, mice were treated preoperatively with terutroban $(30 \mathrm{mg} / \mathrm{kg}), \mathrm{U}-46619(2 \mu \mathrm{g} /$ $\mathrm{kg}$ ), or clopidogrel $(10 \mathrm{mg} / \mathrm{kg})$. For T cell depletion, anti-mouse CD4 (clone GK1.5), anti-mouse CD8 (clone 53-6.72), or isotype control (rat IgG2b) obtained from Bio X Cell was utilized (200 $\mu \mathrm{g} /$ mouse q 3 days, i.p.). For $\mathrm{PGE}_{2}$ studies, mice were treated preoperatively with ketorolac and a $\mathrm{PGE}_{2}$-neutralizing antibody (Cayman Chemical) (200 $\mu \mathrm{g} /$ mouse q 3 days) or isotype control (rat IgG2b). Resolvins (RvD2, RvD3, or RvD4) (15 ng/d/mouse) were administered via miniosmotic pump (Alzet Inc.) 2 hours prior to tumor resection or laparotomy. Meloxicam SR was administered after surgery per IACUC-approved protocol.

Laparotomy. C57BL/6J or RAG1-KO male mice (Jackson Laboratory) were injected with $10^{4} \mathrm{LLC}, 10^{3} \mathrm{~B} 16 \mathrm{~F} 10$, or $10^{4} \mathrm{EL} 4$ cells and subjected to laparotomy under isoflurane inhalation anesthesia. Bowels were removed, placed on a sterile sheet for 4 minutes, and returned to the peritoneum. The skin was sutured with 4-0 chromic gut absorbable sutures. Tumor size was measured by caliper (width ${ }^{2} \times$ length $\times$ $\left.0.52=\mathrm{mm}^{3}\right)$.

Chemotherapy-stimulated cancer. For dormancy models, $10^{4}$ LLC, EL4, or CT26 cells (ATCC) were subcutaneously injected into male C57BL/6J (LLC and EL4) or BALB/cJ (CT26) mice. Cisplatin $(5 \mathrm{mg} / \mathrm{kg}$ q 5 days), vincristine ( $1 \mathrm{mg} / \mathrm{kg} \mathrm{q} 7$ days), or 5 -FU treatment $(30 \mathrm{mg} / \mathrm{kg}$ $\mathrm{q} 3$ days) was initiated on the day of tumor cell injection. For combination therapies, ketorolac $(7.5 \mathrm{mg} / \mathrm{kg})$ was administered the day before, day of, and day after cisplatin or vincristine as well as the day before and day of 5-FU treatment.

Tumor rechallenge. Preoperative ketorolac- or RvD2-treated mice that survived 90 days after LLC resection and naive age-matched mice were challenged with $10^{6}$ LLC via subcutaneous injection. On day 90 after LLC rechallenge, the LLC tumor-resected mice treated with preoperative ketorolac or RvD2, which did not grow tumors, were subcutaneously injected with $10^{6}$ B16F10.

Preoperative ketorolac-treated LLC tumor-bearing mice $\left(10^{4}\right.$ cells) were subjected to a laparotomy. Ninety days after laparotomy, these mice and naive age-matched mice were challenged with $10^{6} \mathrm{LLC}$ via subcutaneous injection. On day 90 after LLC rechallenge, the preoperative ketorolac-treated mice subjected to laparotomy, which did not grow tumors, were subcutaneously injected with $10^{6} \mathrm{~B} 16 \mathrm{~F} 10$.
Adoptive transfer. Splenocytes were obtained as follows: spleens from preoperative ketorolac-treated mice after LLC resection or agematched naive $\mathrm{C} 57 \mathrm{BL} / 6 \mathrm{~J}$ mice were dissociated and filtered through $40 \mu \mathrm{m}$ strainers. CD $8 \alpha^{+} \mathrm{T}$ cells were isolated from this population by magnetic selection using a mouse $\mathrm{CD} 8 \alpha^{+} \mathrm{T}$ Cell Isolation Kit (Miltenyi Biotec). Splenocytes or isolated CD $8 \alpha^{+} \mathrm{T}$ cells were stained with CD $8 \alpha$ APC-conjugated antibody (clone 53-6.7; R\&D systems) and CD3 FITC-conjugated antibody (clone 17A2; BioLegend). Staining was assessed via BD LSR Fortessa (BD Biosciences) at the DFCI Cytometry Core and analyzed using FlowJo software (Tree Star Inc.). Splenocytes $\left(6 \times 10^{7}\right.$ cells $/$ mouse $)$ or isolated $\mathrm{CD} 8 \alpha^{+} \mathrm{T}$ cells $\left(3 \times 10^{6}\right.$ cells/ mouse) were intravenously injected into $\mathrm{C} 57 \mathrm{BL} / 6 \mathrm{~J}$ mice.

Immunohistochemistry. Slides were incubated with $\mathrm{FOXP}^{+}, \mathrm{CD}^{+}$, or $\mathrm{CD}^{+}$(Abcam; $\left.1: 100\right)$ at $4^{\circ} \mathrm{C}$ overnight. TSA Biotin (PerkinElmer) was used for signal amplification. The Zeiss A1 Scope, Axiocam ICc5, and Zeiss efficient navigation software (Zeiss) were used for imaging slides (10-20 fields/tumor). Immune cells per field were quantified via ImageJ.

LC-MS/MS. Plasma from preoperative ketorolac-treated and control mice after LLC resection was analyzed as described (99), with a Shimadzu LC-20AD HPLC and a Shimadzu SIL-20AC autoinjector (Shimadzu Corp.) paired with a QTrap 6500 (ABSciex).

scRNA-Seq analysis. Spleens from control and preoperative ketorolac-treated mice at 7 days after LLC resection were processed using the $10 \times$ genomics approach. Single-cell libraries were prepared using the Chromium 3' reagent kit v2 and sequenced using NextSeq 500 platform to generate approximately 50,000 reads/cell. scRNA-Seq data after standard quality control was aligned to the reference genome (mm10) using the 10× Cell Ranger pipeline. Preprocessed and filtered normalized data were subjected to unsupervised analysis using PCA (Seurat v2.0 Bioconductor package; ref. 100) to identify principal components with significant variation that was used as input for tSNE analysis (101) to determine overall relationship among cells. Cells with similar transcriptome profiles were clustered together, and the clusters were subsequently annotated to different cell types based on expression of specific transcripts, e.g., T cells $\left(\mathrm{Cd} 3 \mathrm{~d}^{+}\right), \mathrm{B}$ cells $\left(\mathrm{Cd} 19^{+}\right.$,

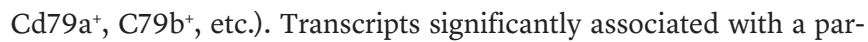
ticular cell type were identified by comparing the expression profile of the target cell with the rest of the cells using nonparametric Wilcox's rank test $(P<0.01)$ and fold change $(>1.2)$.

Identification of treatment-altered genes and pathways in specific cell types. To determine the effect of treatment on specific cell types, comparative analysis of cell abundance was performed on the control and ketorolac-treated samples. Comparative analysis of the transcriptome profiles of each cell type between ketorolac-treated and control groups was performed using a linear model for microarray analysis approach in R language (102). Pathways/Functions enrichment and systems biology analyses using the Ingenuity Pathway Analysis software package (IPA 9.0; QIAGEN) were performed on transcripts that were significantly associated with different $\mathrm{T}$ cell and granulocyte clusters or differentially expressed in the same cell types between treatment and control groups.

Statistic. For in vivo experiments, Student's $t$ test and ANOVA were utilized. Student's $t$ test was utilized to evaluate significance of in vitro experiments. The Kolmogorov-Smirnov test was used to evaluate the assumption of normality of continuous variables, and no significant departures from normality were detected. Summary data are reported as mean \pm SEM. Longitudinal tumor growth data were analyzed using 2-factor repeated-measures mixed effects ANOVA with 
the Greenhouse-Geisser F-test to assess overall group differences followed by Tukey's post hoc comparisons, where treatment was considered the between-subjects factor and serial tumor measurements the within-subjects factor. One-way ANOVA was used to compare the groups with respect to cytokines and biomarker variables. Survival after tumor resection was analyzed using the Kaplan-Meier productlimit model with the log-rank test to evaluate survival differences over time between treatment groups. $P$ values of less than 0.05 were considered statistically significant.

Study approval. Animal studies were approved by the Institutional Animal Care and Use Committee at Beth Israel Deaconess Medical Center.

\section{Author contributions}

DP, MWK, SH, MB, VS, CNS, and VPS designed research. DP, JY, AG, MMG, MLS, SSB, DRB, JC, BAS, JP, HY, AF, SJS, DSF, and MB performed research. MAS and $\mathrm{BDH}$ contributed reagents and analytic tools. DP, SJS, MB, CNS, and VPS analyzed data. DP, MB, AG, MMG, MWK, SH, and VPS wrote the paper.

\section{Acknowledgments}

We gratefully acknowledge Steve Moskowitz (Advanced Medical Graphics) for photography and preparation of the figures and thank Kristen Lehner, Donna Vatnick, and Chantal Barksdale for excellent technical assistance. This work was supported by Nation- al Cancer Institute grants RO1 01CA170549 (to DP and CNS), ROCA148633 (to DP), and R01GM038765 (to CNS); Beth Israel Deaconess Medical Center seed funds and a gift from the Sheth family (to VPS); the Credit Unions Kids at Heart Team (to DP); the Stop and Shop Pediatric Brain Tumor Fund (to MWK); the C.J. Buckley Pediatric Brain Tumor Fund (to MWK); Alex's Lemonade Stand (to MWK); Molly's Magic Wand for Pediatric Brain Tumors (to MWK); the Markoff Foundation Art-In-Giving Foundation (to MWK); the Kamen Foundation (to MWK); Jared Branfman Sunflowers for Life (to MWK); the Joe Andruzzi Foundation (to MWK); National Institute of Environmental Health Science Superfund Research Program grant P42 ES004699, and National Institute of Environmental Health Science grant RO1 ESO02710 (to BDH).

Address correspondence to: Dipak Panigrahy, 99 Brookline Avenue, RN220, Boston, Massachusetts 02215, USA. Phone: 617.667.8202; Email: dpanigra@bidmc.harvard.edu. Or to Charles N. Serhan, 60 Fenwood Road, Room 3012, Boston, Massachusetts 02115, USA. Phone: 617.525.5001; Email: cserhan@bwh. harvard.edu. Or to Vikas P. Sukhatme, 100 Woodruff Circle, Suite 423, Atlanta, Georgia 30322, USA. Phone: 404.727.5631; Email: vsukhatme@emory.edu.

MWK's present address is: Bristol-Myers Squibb, Lawrenceville, New Jersey, USA.
1. Fisher B, Fisher ER. Experimental evidence in support of the dormant tumor cell. Science. 1959;130(3380):918-919.

2. Krall JA, et al. The systemic response to surgery triggers the outgrowth of distant immune-controlled tumors in mouse models of dormancy. Sci Transl Med. 2018;10(436):eaan3464.

3. Ananth AA, et al. Surgical stress abrogates pre-existing protective $\mathrm{T}$ cell mediated antitumor immunity leading to postoperative cancer recurrence. PLoS ONE. 2016;11(5):e0155947.

4. Lee JW, et al. Surgical stress promotes tumor growth in ovarian carcinoma. Clin Cancer Res. 2009;15(8):2695-2702.

5 . Antonio $\mathrm{N}$, et al. The wound inflammatory response exacerbates growth of pre-neoplastic cells and progression to cancer. EMBO J. 2015;34(17):2219-2236.

6. Mathenge EG, et al. Core needle biopsy of breast cancer tumors increases distant metastases in a mouse model. Neoplasia. 2014;16(11):950-960.

7. Alieva M, et al. Preventing inflammation inhibits biopsy-mediated changes in tumor cell behavior. Sci Rep. 2017;7(1):7529.

8. Hobson J, et al. Acute inflammation induced by the biopsy of mouse mammary tumors promotes the development of metastasis. Breast Cancer Res Treat. 2013;139(2):391-401.

9. Sulciner ML, et al. Resolvins suppress tumor growth and enhance cancer therapy. JExp Med. 2018;215(1):115-140.

10. Gartung A, et al. Suppression of chemotherapyinduced cytokine/lipid mediator surge and ovarian cancer by a dual COX-2/sEH inhibitor. Proc Natl Acad Sci U S A. 2019;116(5):1698-1703.

11. Retsky M, Demicheli R, Hrushesky W, Baum
M, Gukas I. Surgery triggers outgrowth of latent distant disease in breast cancer: an inconvenient truth? Cancers (Basel). 2010;2(2):305-337.

12. Serhan CN. Pro-resolving lipid mediators are leads for resolution physiology. Nature. 2014;510(7503):92-101.

13. Cata JP, et al. Inflammation and pro-resolution inflammation after hepatobiliary surgery. World J Surg Oncol. 2017;15(1):152

14. Révész L. Effect of tumour cells killed by x-rays upon the growth of admixed viable cells. Nature. 1956;178(4547):1391-1392.

15. Shakhar G, Ben-Eliyahu S. Potential prophylactic measures against postoperative immunosuppression: could they reduce recurrence rates in oncological patients? Ann Surg Oncol. 2003;10(8):972-992.

16. Forget P, Simonet O, De Kock M. Cancer surgery induces inflammation, immunosuppression and neo-angiogenesis, but is it influenced by analgesics? F100ORes. 2013;2:102.

17. Chiang N, Schwab JM, Fredman G, Kasuga K, Gelman S, Serhan CN. Anesthetics impact the resolution of inflammation. PLOS ONE 2008;3(4):e1879.

18. Chang J, et al. Chemotherapy-generated cell debris stimulates colon carcinoma tumor growth via osteopontin. FASEB J. 2019;33(1):114-125.

19. Endo S, et al. Preoperative chemotherapy increases cytokine production after lung cancer surgery. Eur J Cardiothorac Surg. 2004;26(4):787-791.

20. Lee Y, Kollara A, May T, Brown TJ. Wounding promotes ovarian cancer progression and decreases efficacy of cisplatin in a syngeneic mouse model. JOvarian Res. 2018;11(1):56.

21. Gough MJ, et al. Adjuvant therapy with agonistic antibodies to CD134 (OX40) increases local control after surgical or radiation therapy of cancer in mice. JImmunother. 2010;33(8):798-809.

22. Avraham R, Benish M, Inbar S, Bartal I, Rosenne E, Ben-Eliyahu S. Synergism between immunostimulation and prevention of surgery-induced immune suppression: an approach to reduce post-operative tumor progression. Brain Behav Immun. 2010;24(6):952-958.

23. Tai LH, Auer R. Attacking postoperative metastases using perioperative oncolytic viruses and viral vaccines. Front Oncol. 2014;4:217.

24. Park CG, Hartl CA, Schmid D, Carmona EM, Kim HJ, Goldberg MS. Extended release of perioperative immunotherapy prevents tumor recurrence and eliminates metastases. Sci Transl Med. 2018;10(433):pii: eaar1916.

25. Gough MJ, Baird JR, Bell RB. Implantable biomaterials to provide local immunotherapy following surgical resection. Oncotarget. 2018;9(102):37612-37613.

26. Horowitz M, Neeman E, Sharon E, Ben-Eliyahu S. Exploiting the critical perioperative period to improve long-term cancer outcomes. Nat Rev Clin Oncol. 2015;12(4):213-226.

27. Hiller JG, Perry NJ, Poulogiannis G, Riedel B, Sloan EK. Perioperative events influence cancer recurrence risk after surgery. Nat Rev Clin Oncol. 2018;15(4):205-218.

28. Demicheli R, Terenziani M, Valagussa P, Moliterni A, Zambetti M, Bonadonna G. Local recurrences following mastectomy: support for the concept of tumor dormancy. J Natl Cancer Inst. 1994;86(1):45-48.

29. Retsky MW, Demicheli R, Hrushesky WJ, Baum M, Gukas ID. Dormancy and surgery-driven escape 
from dormancy help explain some clinical features of breast cancer. APMIS. 2008;116(7-8):730-741.

30. Dillekås H, Demicheli R, Ardoino I, Jensen SAH, Biganzoli E, Straume O. The recurrence pattern following delayed breast reconstruction after mastectomy for breast cancer suggests a systemic effect of surgery on occult dormant micrometastases. Breast Cancer Res Treat. 2016;158(1):169-178.

31. de Boer M, et al. Micrometastases or isolated tumor cells and the outcome of breast cancer. N Engl JMed. 2009;361(7):653-663.

32. Folkman J. Angiogenesis: an organizing principle for drug discovery? Nat Rev Drug Discov. 2007;6(4):273-286.

33. Bovill JG. Surgery for cancer: does anesthesia matter? Anesth Analg. 2010;110(6):1524-1526.

34. Forget $P$, et al. Do intraoperative analgesics influence breast cancer recurrence after mastectomy? A retrospective analysis. Anesth Analg. 2010;110(6):1630-1635.

35. Ahn HJ, Kim MH. The effects of ketorolac on T cell subsets in patients undergoing a gastrectomy. Korean JAnesthesiol. 2001;40 (2):201-209.

36. Wang D, DuBois RN. Role of prostanoids in gastrointestinal cancer. J Clin Invest. 2018;128(7):2732-2742.

37. Howe LR, Dannenberg AJ. A role for cyclooxygenase-2 inhibitors in the prevention and treatment of cancer. Semin Oncol. 2002;29(3 Suppl 11):111-119.

38. Wang D, Dubois RN. Eicosanoids and cancer. Nat Rev Cancer. 2010;10(3):181-193.

39. Gilroy DW, Colville-Nash PR, Willis D, Chivers J, Paul-Clark MJ, Willoughby DA. Inducible cyclooxygenase may have anti-inflammatory properties. Nat Med.1999;5(6):698-701.

40. Levy BD, Clish CB, Schmidt B, Gronert K, Serhan $\mathrm{CN}$. Lipid mediator class switching during acute inflammation: signals in resolution. Nat Immunol. 2001;2(7):612-619.

41. Serhan CN, Clish CB, Brannon J, Colgan SP, Chiang N, Gronert K. Novel functional sets of lipid-derived mediators with antiinflammatory actions generated from omega- 3 fatty acids via cyclooxygenase 2-nonsteroidal antiinflammatory drugs and transcellular processing. J Exp Med. 2000;192(8):1197-1204.

42. Hangai $S$, et al. PGE2 induced in and released by dying cells functions as an inhibitory DAMP. Proc Natl Acad Sci U S A. 2016;113(14):3844-3849.

43. Serhan CN, Levy BD. Resolvins in inflammation: emergence of the pro-resolving superfamily of mediators. J Clin Invest. 2018;128(7):2657-2669.

44. Chan AT, Giovannucci EL, Meyerhardt JA, Schernhammer ES, Curhan GC, Fuchs CS. Long-term use of aspirin and nonsteroidal antiinflammatory drugs and risk of colorectal cancer. JAMA. 2005;294(8):914-923.

45. Serhan CN, et al. Resolvins: a family of bioactive products of omega-3 fatty acid transformation circuits initiated by aspirin treatment that counter proinflammation signals. J Exp Med. 2002;196(8):1025-1037.

46. Dalli J, et al. Resolvin D3 and aspirin-triggered resolvin D3 are potent immunoresolvents. Chem Biol. 2013;20(2):188-201.

47. Winkler JW, et al. Resolvin D4 stereoassignment and its novel actions in host protection and bac- terial clearance. Sci Rep. 2016;6:18972.

48. Ye Y, et al. Anti-cancer and analgesic effects of resolvin D2 in oral squamous cell carcinoma. Neuropharmacology. 2018;139:182-193.

49. Kuang H, Hua X, Zhou J, Yang R. Resolvin D1 and E1 alleviate the progress of hepatitis toward liver cancer in long-term concanavalin A-induced mice through inhibition of NF- $\mathrm{\kappa B}$ activity. Oncol Rep. 2016;35(1):307-317.

50. Zong L, et al. Lipoxin A4 reverses mesenchymal phenotypes to attenuate invasion and metastasis via the inhibition of autocrine TGF- $\beta 1$ signaling in pancreatic cancer. J Exp Clin Cancer Res. 2017;36(1):181.

51. Gilligan MM, et al. Aspirin-triggered proresolving mediators stimulate resolution in cancer. Proc Natl Acad Sci U S A. 2019;116(13):6292-6297.

52. O'Reilly MS, et al. Angiostatin: a novel angiogenesis inhibitor that mediates the suppression of metastases by a Lewis lung carcinoma. Cell. 1994;79(2):315-328.

53. Panigrahy D, et al. Epoxyeicosanoids stimulate multiorgan metastasis and tumor dormancy escape in mice. J Clin Invest. 2012;122(1):178-191.

54. Jia X, et al. Emodin suppresses pulmonary metastasis of breast cancer accompanied with decreased macrophage recruitment and M2 polarization in the lungs. Breast Cancer Res Treat. 2014;148(2):291-302.

55. Coffey JC, Wang JH, Smith MJ, Bouchier-Hayes D, Cotter TG, Redmond HP. Excisional surgery for cancer cure: therapy at a cost. Lancet Oncol. 2003;4(12):760-768.

56. Goss PE, Chambers AF. Does tumour dormancy offer a therapeutic target? Nat Rev Cancer. 2010;10(12):871-877.

57. Warner TD, Giuliano F, Vojnovic I, Bukasa A, Mitchell JA, Vane JR. Nonsteroid drug selectivities for cyclo-oxygenase-1 rather than cyclooxygenase- 2 are associated with human gastrointestinal toxicity: a full in vitro analysis. Proc Natl Acad Sci U S A. 1999;96(13):7563-7568.

58. Waterbury LD, Silliman D, Jolas T. Comparison of cyclooxygenase inhibitory activity and ocular anti-inflammatory effects of ketorolac tromethamine and bromfenac sodium. Curr Med Res Opin. 2006;22(6):1133-1140.

59. Vitale P, Panella A, Scilimati A, Perrone MG. COX-1 inhibitors: beyond structure toward therapy. Med Res Rev. 2016;36(4):641-671.

60. Guillem-Llobat P, et al. Aspirin prevents colorectal cancer metastasis in mice by splitting the crosstalk between platelets and tumor cells. Oncotarget. 2016;7(22):32462-32477.

61. Lucotti S, et al. Aspirin blocks formation of metastatic intravascular niches by inhibiting platelet-derived COX-1/thromboxane A2. JClin Invest. 2019;129(5):1845-1862.

62. Schnittert J, Heinrich MA, Kuninty PR, Storm G, Prakash J. Reprogramming tumor stroma using an endogenous lipid lipoxin A4 to treat pancreatic cancer. Cancer Lett. 2018;420:247-258.

63. Dittmer J. Mechanisms governing metastatic dormancy in breast cancer. Semin Cancer Biol. 2017;44:72-82.

64. Manjili MH. The inherent premise of immunotherapy for cancer dormancy. Cancer Res. 2014;74(23):6745-6749.
65. Fisher B, Gunduz N, Saffer EA. Influence of the interval between primary tumor removal and chemotherapy on kinetics and growth of metastases. Cancer Res. 1983;43(4):1488-1492.

66. Tewari KS, Java JJ, Eskander RN, Monk BJ, Burger RA. Early initiation of chemotherapy following complete resection of advanced ovarian cancer associated with improved survival: NRG Oncology/Gynecologic Oncology Group study. Ann Oncol. 2016;27(1):114-121.

67. Brodin P, Davis MM. Human immune system variation. Nat Rev Immunol. 2017;17(1):21-29.

68. Krämer A, Green J, Pollard J, Tugendreich S. Causal analysis approaches in Ingenuity Pathway Analysis. Bioinformatics. 2014;30(4):523-530.

69. Mansfield AS, et al. Regional immunity in melanoma: immunosuppressive changes precede nodal metastasis. Mod Pathol. 2011;24(4):487-494.

70. Zhou X, Yu S, Zhao DM, Harty JT, Badovinac VP, Xue HH. Differentiation and persistence of memory CD8(+) T cells depend on $\mathrm{T}$ cell factor 1. Immunity. 2010;33(2):229-240.

71. Shao L, Zhang B, Wang L, Wu L, Kan Q, Fan K. MMP-9-cleaved osteopontin isoform mediates tumor immune escape by inducing expansion of myeloid-derived suppressor cells. Biochem Biophys Res Commun. 2017;493(4):1478-1484.

72. Takada Y, Bhardwaj A, Potdar P, Aggarwal BB. Nonsteroidal anti-inflammatory agents differ in their ability to suppress NF-kappaB activation, inhibition of expression of cyclooxygenase- 2 and cyclin D1, and abrogation of tumor cell proliferation. Oncogene. 2004;23(57):9247-9258.

73. $\mathrm{Hu} \mathrm{H}$, et al. Phosphoinositide 3-kinase regulates glycolysis through mobilization of aldolase from the actin cytoskeleton. Cell. 2016;164(3):433-446.

74. Borregaard N, Herlin T. Energy metabolism of human neutrophils during phagocytosis. JClin Invest. 1982;70(3):550-557.

75. Martin KJ, Muessel MJ, Pullar CE, Willars GB, Wardlaw AJ. The role of phosphoinositide 3-kinases in neutrophil migration in 3D collagen gels. PLOS ONE. 2015;10(2):e0116250.

76. Hou Z, Falcone DJ, Subbaramaiah K, Dannenberg AJ. Macrophages induce COX-2 expression in breast cancer cells: role of IL-1 $\beta$ autoamplification. Carcinogenesis. 2011;32(5):695-702.

77. Flamiatos JF, et al. Cyclooxygenase-2 (COX-2) inhibition for prostate cancer chemoprevention: double-blind randomised study of pre-prostatectomy celecoxib or placebo. BJU Int . 2017;119(5):709-716.

78. Zhou YY, Hu ZG, Zeng FJ, Han J. Clinical profile of cyclooxygenase-2 inhibitors in treating non-small cell lung cancer: a meta-analysis of nine randomized clinical trials. PLoS One. 2016;11(3):e0151939.

79. Edelman MJ, et al. Randomized, double-blind, placebo-controlled, multicenter phase II study of the efficacy and safety of apricoxib in combination with either docetaxel or pemetrexed in patients with biomarker-selected non-small-cell lung cancer. J Clin Oncol. 2015;33(2):189-194.

80. Csiki I, et al. Targeting cyclooxygenase- 2 in recurrent non-small cell lung cancer: a phase II trial of celecoxib and docetaxel. Clin Cancer Res. 2005;11(18):6634-6640.

81. Groen HJ, et al. Randomized, placebo-controlled phase III study of docetaxel plus carboplatin with 
celecoxib and cyclooxygenase-2 expression as a biomarker for patients with advanced non-smallcell lung cancer: the NVALT-4 study. JClin Oncol. 2011;29(32):4320-4326.

82. Vinogradova Y, Coupland C, Hippisley-Cox J. Exposure to cyclooxygenase-2 inhibitors and risk of cancer: nested case-control studies. Br JCancer. 2011;105(3):452-459.

83. Keller JJ, et al. Molecular analysis of sulindacresistant adenomas in familial adenomatous polyposis. Clin Cancer Res. 2001;7(12):4000-4007.

84. Seiz M, et al. Far-distant metastases along the CSF pathway of glioblastoma multiforme during continuous low-dose chemotherapy with temozolomide and celecoxib. Neurosurg Rev. 2010;33(3):375-381; discussion 381.

85. Zelenay S, et al. Cyclooxygenase-dependent tumor growth through evasion of immunity. Cell. 2015;162(6):1257-1270.

86. Loo TM, et al. Gut microbiota promotes obesityassociated liver cancer through PGE. Cancer Discov. 2017;7(5):522-538.

87. Mao JT, et al. Chemoprevention strategies with cyclooxygenase-2 inhibitors for lung cancer. Clin Lung Cancer. 2005;7(1):30-39.

88. Baratelli F, et al. PGE2 confers survivindependent apoptosis resistance in human monocyte-derived dendritic cells. J Leukoc Biol. 2005;78(2):555-564.

89. Theisen E, et al. Cyclooxygenase-1 and -2 play contrasting roles in Listeria-stimulated immunity. J Immunol. 2018;200(11):3729-3738.

90. Ekambaram P, Lambiv W, Cazzolli R, Ashton AW, Honn KV. The thromboxane synthase and receptor signaling pathway in cancer: an emerging paradigm in cancer progression and metastasis. Cancer Metastasis Rev. 2011;30(3-4):397-408.

91. Servais L, et al. Platelets contribute to the initiation of colitis-associated cancer by promoting immunosuppression. J Thromb Haemost. 2018;16(4):762-777.

92. Afek A, et al. Clopidogrel attenuates atheroma formation and induces a stable plaque phenotype in apolipoprotein E knockout mice. Microvasc Res. 2009;77(3):364-369.

93. Lannan KL, Spinelli SL, Blumberg N, Phipps RP. Maresin 1 induces a novel pro-resolving phenotype in human platelets. J Thromb Haemost. 2017;15(4):802-813.

94. Eggermont AM, et al. Prolonged survival in stage III melanoma with ipilimumab adjuvant therapy. N Engl JMed. 2016;375(19):1845-1855.

95. Karagiannis GS, et al. Neoadjuvant chemotherapy induces breast cancer metastasis through a
TMEM-mediated mechanism. Sci Transl Med. 2017;9(397):eaan0026.

96. Volk-Draper L, et al. Paclitaxel therapy promotes breast cancer metastasis in a TLR4-dependent manner. Cancer Res. 2014;74(19):5421-5434.

97. Chang YS, Jalgaonkar SP, Middleton JD, Hai T. Stress-inducible gene. Proc Natl Acad Sci U S A. 2017;114(34):E7159-E7168.

98. Aguirre-Ghiso JA. Models, mechanisms and clinical evidence for cancer dormancy. Nat Rev Cancer. 2007;7(11):834-846.

99. Yang J, Schmelzer K, Georgi K, Hammock BD. Quantitative profiling method for oxylipin metabolome by liquid chromatography electrospray ionization tandem mass spectrometry. Anal Chem. 2009;81(19):8085-8093.

100.Butler A, Hoffman P, Smibert P, Papalexi E, Satija R. Integrating single-cell transcriptomic data across different conditions, technologies, and species. Nat Biotechnol. 2018;36(5):411-420.

101.Van der Maaten L, Hinton GE. Visualizing high-dimensional data using tSNE.J Mach Learn Res. 2008;9(Nov):2579-2605.

102. Ritchie ME, et al. limma powers differential expression analyses for RNA-sequencing and microarray studies. Nucleic Acids Res. 2015;43(7):e47. 